\title{
Best constants for some operators associated with the Fourier and Hilbert transforms
}

\author{
by
}

\author{
B. Hollenbeck (Emporia, KS), N. J. Kalton (Columbia, MO) \\ and I. E. VERBitsky (Columbia, MO)
}

\begin{abstract}
We determine the norm in $L^{p}\left(\mathbb{R}_{+}\right), 1<p<\infty$, of the operator $I-\mathcal{F}_{\mathrm{s}} \mathcal{F}_{\mathrm{c}}$, where $\mathcal{F}_{\mathrm{c}}$ and $\mathcal{F}_{\mathrm{S}}$ are respectively the cosine and sine Fourier transforms on the positive real axis, and $I$ is the identity operator. This solves a problem posed in 1984 by M. S. Birman [Bir] which originated in scattering theory for unbounded obstacles in the plane.

We also obtain the $L^{p}$-norms of the operators $a I+b H$, where $H$ is the Hilbert transform (conjugate function operator) on the circle or real line, for arbitrary real $a, b$. Best constants in other related inequalities are found.

In a more general framework, we present an alternative proof of the important theorem of Cole relating best constant inequalities involving the Hilbert transform and the existence of subharmonic minorants, which extends to several variables and plurisubharmonic minorants.
\end{abstract}

1. Introduction. Let $\mathcal{F}_{\mathrm{c}}$ and $\mathcal{F}_{\mathrm{s}}$ denote respectively the cosine and sine Fourier transforms on the positive real axis $\mathbb{R}_{+}=(0, \infty)$ :

$$
\mathcal{F}_{\mathrm{c}} u(x)=\sqrt{\frac{2}{\pi}} \int_{\mathbb{R}_{+}} u(t) \cos t x d t, \quad \mathcal{F}_{\mathrm{s}} u(x)=\sqrt{\frac{2}{\pi}} \int_{\mathbb{R}_{+}} u(t) \sin t x d t .
$$

Both $\mathcal{F}_{\mathrm{c}}$ and $\mathcal{F}_{\mathrm{s}}$ are unitary, self-adjoint operators on $L^{2}\left(\mathbb{R}_{+}\right)$.

The so-called re-expansion operator is defined by $\Pi=\mathcal{F}_{\mathrm{s}} \mathcal{F}_{\mathrm{c}}$, and its adjoint by $\Pi^{*}=\mathcal{F}_{\mathrm{c}} \mathcal{F}_{\mathrm{s}}$. A direct calculation yields

$$
\|I-\Pi\|_{L^{2}\left(\mathbb{R}_{+}\right)}=\left\|I-\Pi^{*}\right\|_{L^{2}\left(\mathbb{R}_{+}\right)}=\left\|\mathcal{F}_{\mathrm{c}}-\mathcal{F}_{\mathrm{s}}\right\|_{L^{2}\left(\mathbb{R}_{+}\right)}=\sqrt{2}
$$

It is not difficult to see that $\Pi$ can be extended to a bounded operator on $L^{p}\left(\mathbb{R}_{+}\right)$for $1<p<\infty$.

One of the motivations for the present paper is to answer the question, stated by M. S. Birman as Problem 1 in [Bir], on the exact value of the

2000 Mathematics Subject Classification: Primary 42B10; Secondary 46E30.

The first and third authors were supported in part by NSF Grant DMS-0070623.

The second author was supported in part by NSF Grant DMS-9870027. 
operator norm $\|I-\Pi\|_{L^{p}\left(\mathbb{R}_{+}\right)}, 1<p<\infty$. Along with other problems posed in [Bir], it has its origin in scattering by unbounded obstacles in the plane. In particular, a periodic analogue of $I-\mathcal{F}_{\mathrm{s}} \mathcal{F}_{\mathrm{c}}$ serves as the scattering matrix for diffraction by a semi-infinite screen (see [I1], [I2]).

The re-expansion operator $\Pi=\mathcal{F}_{\mathrm{s}} \mathcal{F}_{\mathrm{c}}$ appears naturally in the following model problem considered in [Bir].

For a pair $\mathcal{H}_{0}, \mathcal{H}$ of self-adjoint operators on Hilbert space, the wave operators $W_{ \pm}$are defined by

$$
W_{ \pm}\left(\mathcal{H}, \mathcal{H}_{0}\right)=\lim _{t \rightarrow \pm \infty} e^{i t \mathcal{H}} e^{-i t \mathcal{H}_{0}}
$$

where the limit is understood in the sense of strong operator convergence.

Now let $\mathcal{H}_{0}$ and $\mathcal{H}$ denote the operator $-d^{2} / d x^{2}$ on $\mathbb{R}_{+}$with the boundary conditions $u^{\prime}(0)=0$ and $u(0)=0$, respectively. Then, as shown in [Bir],

$$
W_{ \pm}\left(\mathcal{H}, \mathcal{H}_{0}\right)= \pm i \Pi \text {. }
$$

Re-expansion operators also arise in polar decompositions of classical differential operators, and other problems of mathematical physics.

To extend $\Pi$ and $\Pi^{*}$ to bounded operators on $L^{p}\left(\mathbb{R}_{+}\right), 1<p<\infty$, notice that they can be defined as singular integral operators:

$$
\Pi u(x)=\frac{1}{\pi} \text { p.v. } \int_{\mathbb{R}_{+}} \frac{2 x u(t)}{x^{2}-t^{2}} d t, \quad \Pi^{*} u(x)=\frac{1}{\pi} \text { p.v. } \int_{\mathbb{R}_{+}} \frac{2 t u(t)}{t^{2}-x^{2}} d t, \quad x \in \mathbb{R}_{+} .
$$

This follows by looking at the restrictions of the operator $H^{\mathbb{R}} \Sigma$ to the subspaces $L_{\text {odd }}^{p}(\mathbb{R})$ and $L_{\text {even }}^{p}(\mathbb{R})$ consisting of odd and even functions respectively. Here $\Sigma$ is the multiplication by $\operatorname{sign} x$, and $H^{\mathbb{R}}$ is the Hilbert transform on the real line defined by

$$
H^{\mathbb{R}} u(x)=\frac{1}{\pi} \text { p.v. } \int_{\mathbb{R}} \frac{u(t)}{x-t} d t, \quad x \in \mathbb{R} .
$$

In other words, $\mathcal{F}_{\mathrm{s}} \mathcal{F}_{\mathrm{c}}=H+H_{1}$, and $\mathcal{F}_{\mathrm{c}} \mathcal{F}_{\mathrm{s}}=-H+H_{1}$, where

$$
H u(x)=\frac{1}{\pi} \text { p.v. } \int_{\mathbb{R}_{+}} \frac{u(t)}{x-t} d t, \quad H_{1} u(x)=\frac{1}{\pi} \int_{\mathbb{R}_{+}} \frac{u(t)}{x+t} d t, \quad x \in \mathbb{R}_{+},
$$

are the Hilbert transforms on $\mathbb{R}_{+}$with kernels $\frac{1}{\pi(x-y)}$ and $\frac{1}{\pi(x+y)}$ respectively. (Sometimes we will use the notation $H^{\mathbb{R}_{+}}$in place of $H$ to distinguish it from the Hilbert transform $H^{\mathbb{R}}$ on the real line.)

It follows from the theory of singular integral operators that the spectrum of $\Pi$ in $L^{p}\left(\mathbb{R}_{+}\right)$, say in the case $p \geq 2$, is the arc of the circle $\left|z-\cot \frac{\pi}{p}\right|=$ $\csc \frac{\pi}{p}$ with the endpoints at $\pm i$ which lies in the right half-plane (see [GK2]). 
Moreover, one can show (Sec. 6) that

$$
\|\Pi\|_{L^{p}\left(\mathbb{R}_{+}\right)}=\cot \frac{\pi}{2 p^{*}}, \quad p^{*}=\max \left(p, p^{\prime}\right),
$$

where $p^{\prime}=p /(p-1)$. Note that $\|\Pi\|_{L^{p}\left(\mathbb{R}_{+}\right)}$coincides with the norm of the Hilbert transform on the real line determined by Gohberg and Krupnik [GK1] for $p=2^{n}(n=2,3, \ldots)$, and later by Pichorides [Pi] and Cole (see [G]) for all $1<p<\infty$.

However, computing $\|I-\Pi\|_{L^{p}\left(\mathbb{R}_{+}\right)}$is substantially more difficult, and requires new ideas. We establish below the following formula which answers Birman's question:

$$
\left\|I-\mathcal{F}_{\mathrm{s}} \mathcal{F}_{\mathrm{c}}\right\|_{L^{p}\left(\mathbb{R}_{+}\right)}=\left\|I-\mathcal{F}_{\mathrm{c}} \mathcal{F}_{\mathrm{s}}\right\|_{L^{p}\left(\mathbb{R}_{+}\right)}=A_{p}
$$

where the constant $A_{p}$ is defined by

$$
A_{p}=\sqrt{2} \max _{0 \leq \theta \leq 2 \pi}\left[\frac{\left|\cos \left(\theta-\frac{\pi}{4}\right)\right|^{p}+\left|\cos \left(\theta-\frac{\pi}{4}+\frac{\pi}{p}\right)\right|^{p}}{|\cos \theta|^{p}+\left|\cos \left(\theta+\frac{\pi}{p}\right)\right|^{p}}\right]^{1 / p}
$$

for all $1<p<\infty$. In particular, it is easy to see that $A_{2}=\sqrt{2}, A_{4}=$ $\sqrt{4+2 \sqrt{5}}$, and $A_{p}=A_{p^{\prime}}$ (the latter is verified using duality).

The proof of the main inequality,

$$
\left\|\mathcal{F}_{\mathrm{s}} f-\mathcal{F}_{\mathrm{c}} f\right\|_{L^{p}\left(\mathbb{R}_{+}\right)} \leq A_{p}\left\|\mathcal{F}_{\mathrm{c}} f\right\|_{L^{p}\left(\mathbb{R}_{+}\right)},
$$

is based on the following estimate obtained in Sec. 4 in a more general setting:

$$
|x-y|^{p} \leq B_{p}|x|^{p}-G(x, y), \quad(x, y) \in \mathbb{R}^{2},
$$

where $B_{p}=A_{p}^{p}$, and $G(x, y)$ is a subharmonic minorant for

$$
\Phi(x, y)=B_{p}|x|^{p}-|x-y|^{p}
$$

in the plane such that $G(0,0)=0$. We actually give an explicit construction for the maximal subharmonic minorant of $\Phi(x, y)$, and at the same time show that the constant $B_{p}$ in the above inequality is sharp.

One of the properties of $G$ used in the proof is

$$
\frac{\partial G}{\partial y}(x, 0)=a_{p} x|x|^{p-2}, \quad a_{p} \geq 0 .
$$

If $p \geq 2$ then actually $a_{p}=p$, which yields a slightly stronger inequality:

$$
\left\|\mathcal{F}_{\mathrm{c}} f-\mathcal{F}_{\mathrm{s}} f\right\|_{L^{p}\left(\mathbb{R}_{+}\right)}^{p}+\|\mathcal{L} f\|_{L^{p}\left(\mathbb{R}_{+}\right)}^{p} \leq A_{p}^{p}\left\|\mathcal{F}_{\mathrm{c}} f\right\|_{L^{p}\left(\mathbb{R}_{+}\right)}^{p}
$$

where

$$
\mathcal{L} f(y)=\sqrt{\frac{2}{\pi}} \int_{0}^{\infty} f(t) e^{-t y} d t, \quad y \geq 0,
$$

is the Laplace transform. 
Notice that a similar problem concerning the norms $\left\|I+\mathcal{F}_{\mathrm{s}} \mathcal{F}_{\mathrm{c}}\right\|_{L^{p}\left(\mathbb{R}_{+}\right)}$ and $\left\|I+\mathcal{F}_{\mathrm{c}} \mathcal{F}_{\mathrm{s}}\right\|_{L^{p}\left(\mathbb{R}_{+}\right)}$demonstrates a certain lack of symmetry. As we will show in Sec. 6,

$$
\left\|I+\mathcal{F}_{\mathrm{s}} \mathcal{F}_{\mathrm{c}}\right\|_{L^{p}\left(\mathbb{R}_{+}\right)}=A_{p}, \quad p \geq 3,
$$

but on the other hand,

$$
\left\|I+\mathcal{F}_{\mathrm{s}} \mathcal{F}_{\mathrm{c}}\right\|_{L^{p}\left(\mathbb{R}_{+}\right)}=1+\tan \frac{\pi}{2 p}>A_{p}, \quad 1<p \leq 2 .
$$

We also find the norm of the operator $a I+b H^{\mathbb{R}}$ in $L^{p}(\mathbb{R})$ for $a, b \in \mathbb{R}$. The following lower estimate for $\left\|a I+b H^{\mathbb{R}}\right\|_{L^{p}(\mathbb{R})}$, which was believed to be sharp for all $a, b \in \mathbb{C}$, is given in [GK2]:

$$
\begin{aligned}
\left\|a I+b H^{\mathbb{R}}\right\|_{L^{p}(\mathbb{R})} \geq & \left(|b|^{2} \cot ^{2} \frac{\pi}{p}+\left(\frac{|a-i b|-|a+i b|}{2}\right)^{2}\right)^{1 / 2} \\
& +\left(|b|^{2} \cot ^{2} \frac{\pi}{p}+\left(\frac{|a-i b|+|a+i b|}{2}\right)^{2}\right)^{1 / 2} .
\end{aligned}
$$

In the case $a=0, b=1$, this estimate is indeed sharp since the right-hand side of the preceding inequality coincides with $\left\|H^{\mathbb{R}}\right\|_{L^{p}(\mathbb{R})}=\cot \frac{\pi}{2 p^{*}}([\mathrm{Pi}]$, $[\mathrm{G}])$. Recently, it was shown in [HV] that equality holds in (1.8) for $a=1$, $b=i$ as well. This gives the norm of the Riesz projection $P_{+}=\frac{1}{2}\left(I+i H^{\mathbb{R}}\right)$ which maps $L^{p}(\mathbb{R})$ onto the Hardy space $H^{p}(\mathbb{R})$; equivalently,

$$
\left\|I+i H^{\mathbb{R}}\right\|_{L^{p}(\mathbb{R})}=2 \csc \frac{\pi}{p} .
$$

Unfortunately, in general (1.8) is not sharp. For $a, b \in \mathbb{R}$, we will show that the actual norm is given by the following formula:

$$
\begin{aligned}
& \left\|a I+b H^{\mathbb{R}}\right\|_{L^{p}(\mathbb{R})} \\
& \quad=\sqrt{a^{2}+b^{2}} \max _{0 \leq \theta \leq 2 \pi}\left[\frac{\left|\cos \left(\theta+\theta_{0}\right)\right|^{p}+\left|\cos \left(\theta+\theta_{0}+\frac{\pi}{p}\right)\right|^{p}}{|\cos \theta|^{p}+\left|\cos \left(\theta+\frac{\pi}{p}\right)\right|^{p}}\right]^{1 / p}
\end{aligned}
$$

where $\theta_{0}=\arctan \frac{b}{a}$. As a corollary, we obtain a uniform estimate for the norms of $(\cos \theta) I+(\sin \theta) H$ (see Theorem 5.6 below):

$$
\max _{0 \leq \theta \leq 2 \pi}\left\|(\cos \theta) I+(\sin \theta) H^{\mathbb{R}}\right\|_{L^{p}(\mathbb{R})}=\cot \frac{\pi}{2 p^{*}} .
$$

We observe that the above formula for $\left\|a I+b H^{\mathbb{R}}\right\|_{L^{p}(\mathbb{R})}$ can be restated as

$$
\left\|a I+b H^{\mathbb{R}}\right\|_{L^{p}(\mathbb{R})}=\|a I+b \mathfrak{h}\|_{l_{2}^{p}}
$$

where the operator $\mathfrak{h}$ is defined by the $2 \times 2$ matrix

$$
\mathfrak{h}=\left(\begin{array}{ll}
\cot \frac{\pi}{p} & -\csc \frac{\pi}{p} \\
\csc \frac{\pi}{p} & -\cot \frac{\pi}{p}
\end{array}\right)
$$

on the space $l_{2}^{p}$, and $\|a I+b \mathfrak{h}\|_{l_{2}^{p}}$ is the corresponding operator norm. 
These results, together with other related facts, are established in Sections 4 and 5. They are derived from a new criterion of the existence of a subharmonic minorant for a $p$-homogeneous function in the complex plane. The proof of this criterion in Sec. 3 is based on a convexity argument, and makes use of the Phragmén-Lindelöf-type theorems for subharmonic functions, and theory of $p$-trigonometrically convex functions [L1], [L2]. It extends and clarifies some earlier results of [E1] and [V].

More general inequalities of this type are discussed in Sec. 5. Here, we draw attention to Theorem 5.3 and Proposition 5.4 where, for example, the estimate

$$
\left\|\left(I+i t H^{\mathbb{T}}\right) u\right\|_{p} \leq\left(1+t^{2} \tan ^{2} \frac{\pi}{2 p}\right)^{1 / 2}\|u\|_{p}, \quad u \in L^{p}(\mathbb{T}),
$$

for $1<p<2$ is shown to hold if $\sqrt{1-p / 3} \leq t<\infty$ but not to hold for every $t>0$. (Here $u$ is assumed to be real-valued.) In fact, in Theorem 5.5 we find a critical value $\tau(p)$ such that this sharp inequality holds for $t \geq \tau(p)$, but fails if $0<t<\tau(p)$. The fact that $\tau(p)$ is a root of a certain nonlinear equation emphasizes that best constant problems of this type can become very complicated. Another example is a solution to Birman's problem given in Sec. 6.

Before giving the proofs of these results, we present in Sec. 2 an alternative proof of a theorem of Cole $[G]$ to the effect that if $F$ is an upper semicontinuous real-valued function on $\mathbb{C}$, the validity of an inequality of the type

$$
\int_{0}^{2 \pi} F \circ f\left(e^{i \theta}\right) \frac{d \theta}{2 \pi} \geq 0
$$

for all polynomials $f$ with $f(0)$ real is equivalent to the existence of a subharmonic minorant $G$ for $F$ with $G(z) \geq 0$ for $z$ real. This result is not strictly needed for the remainder of the paper and the reader may therefore omit this section. It does, however, underscore the importance of the problem of finding subharmonic minorants. The proof we give extends easily to several variables and plurisubharmonic minorants (it is actually based on an argument for a related result in quasi-Banach spaces $[\mathrm{Ka}])\left({ }^{1}\right)$. Note that plurisubharmonic minorants were used in $[\mathrm{HV}]$ to determine the best constant for the Riesz projection.

Other best constant inequalities involving the Hilbert and Fourier transforms, and references to the literature can be found in, e.g., [Ba], [Be], [Bur], [E1], [E2], [ESS], [G], [GK2], [HV], [K], [Li], [Pe], [Pi].

$\left({ }^{1}\right)$ After this paper was initially submitted for publication we learned that another proof for several variables was obtained in $[\mathrm{Po}]$ in a different context. 
We wish to thank the referee for a thorough reading of the manuscript and numerous remarks that improved the exposition.

2. Cole's theorem revisited. In this section we give a discussion of a theorem of Cole, which appeared (with a sketched proof) in [G]. We will give a different and more elementary proof, which extends easily to several variables.

TheOrem 2.1. Suppose $F: \mathbb{C} \rightarrow[-\infty, \infty)$ is an upper semicontinuous function and $E$ is a nonempty subset of $\mathbb{C}$. Then in order that we have the inequality

$$
\int_{0}^{2 \pi} F \circ f\left(e^{i \theta}\right) \frac{d \theta}{2 \pi} \geq 0
$$

whenever $f$ is a polynomial with $f(0) \in E$, it is necessary and sufficient that there exists a subharmonic function $G$ with $G(z) \leq F(z)$ for $z \in \mathbb{C}$ and $G(z) \geq 0$ for $z \in E$.

The proof of Theorem 2.1 depends on the following lemma, which for continuous $F$ is a special case of Lemma 3.1 of [Ka]:

Lemma 2.2. Suppose $F: \mathbb{C} \rightarrow[-\infty, \infty)$ is upper semicontinuous and $f, g$ are polynomials. Then

$$
\begin{aligned}
\limsup _{n \rightarrow \infty} \int_{0}^{2 \pi} F\left(f\left(e^{i \theta}\right)+e^{i n \theta} g\left(e^{i \theta}\right)\right) \frac{d \theta}{2 \pi} & \\
\leq & \int_{0}^{2 \pi} \int_{0}^{2 \pi} F\left(f\left(e^{i \theta}\right)+e^{i \phi} g\left(e^{i \theta}\right)\right) \frac{d \phi}{2 \pi} \frac{d \theta}{2 \pi} .
\end{aligned}
$$

Proof. Using a standard approximation argument it is easy to see that (2.2) follows immediately from the same statement for continuous functions. We thus suppose $F$ is continuous: we will show

$$
\begin{aligned}
\lim _{n \rightarrow \infty} \int_{0}^{2 \pi} F\left(f\left(e^{i \theta}\right)+e^{i n \theta} g\left(e^{i \theta}\right)\right) & \frac{d \theta}{2 \pi} \\
& =\int_{0}^{2 \pi} \int_{0}^{2 \pi} F\left(f\left(e^{i \theta}\right)+e^{i \phi} g\left(e^{i \theta}\right)\right) \frac{d \phi}{2 \pi} \frac{d \theta}{2 \pi} .
\end{aligned}
$$

It suffices by a density argument to show this for functions of the form $F(z)=z^{r} \bar{z}^{s}$ where $r, s=0,1, \ldots$ We have

$$
F\left(f+z^{n} g\right)=\sum_{j=0}^{r} \sum_{k=0}^{s} z^{n j} \bar{z}^{n k}\left(\begin{array}{l}
r \\
j
\end{array}\right)\left(\begin{array}{l}
s \\
k
\end{array}\right) f^{r-j} \bar{f}^{s-k} g^{j} \bar{g}^{k} .
$$


By the Riemann-Lebesgue Lemma the left-hand side of (2.3) coincides with

$$
\sum_{j=0}^{\min (r, s)}\left(\begin{array}{l}
r \\
j
\end{array}\right)\left(\begin{array}{l}
s \\
j
\end{array}\right) \int_{0}^{2 \pi} f\left(e^{i \theta}\right)^{r-j}{\overline{f\left(e^{i \theta}\right)}}^{s-j}\left|g\left(e^{i \theta}\right)\right|^{2 j} \frac{d \theta}{2 \pi} .
$$

However similar reasoning shows that this coincides with the right-hand side of (2.3).

Proof of Theorem 2.1. We will repeatedly use the fact that an upper semicontinuous function is bounded above on any compact set. We need only show that (2.1) implies the existence of $G$. First we show that if $r>0$ and $F$ satisfies $(2.1)$ then

$$
F_{1}(z)=\min \left(F(z), \int_{0}^{2 \pi} F\left(z+r e^{i \theta}\right) \frac{d \theta}{2 \pi}\right)
$$

is also upper semicontinuous and satisfies (2.1). Upper semicontinuity is clear. Now suppose $f, g$ are polynomials with $f(0) \in E$. If $n>0$ then

$$
\int_{0}^{2 \pi} F\left(f\left(e^{i \theta}\right)+e^{i n \theta} g\left(e^{i \theta}\right)\right) \frac{d \theta}{2 \pi} \geq 0 .
$$

Letting $n \rightarrow \infty$ and using Lemma 2.2 yields

$$
\int_{0}^{2 \pi} \int_{0}^{2 \pi} F\left(f\left(e^{i \theta}\right)+e^{i \phi} g\left(e^{i \theta}\right)\right) \frac{d \phi}{2 \pi} \frac{d \theta}{2 \pi} \geq 0 .
$$

Clearly this implies

$$
\int_{0}^{2 \pi} \int_{0}^{2 \pi} F\left(f\left(e^{i \theta}\right)+e^{i \phi}\left|g\left(e^{i \theta}\right)\right|\right) \frac{d \phi}{2 \pi} \frac{d \theta}{2 \pi} \geq 0 .
$$

Let $B$ be a Borel subset of $\mathbb{T}$. Choose polynomials $g_{n}$ so that $\left|g_{n}\right| \leq r$ on $\mathbb{T}$ and

$$
\lim _{n \rightarrow \infty}\left|g_{n}\left(e^{i \theta}\right)\right|=r \chi_{B}\left(e^{i \theta}\right) \quad \text { a.e. }
$$

(This can be achieved, for example, by finding a sequence of outer functions $h_{n} \in H^{\infty}$ with $\left\|h_{n}\right\|_{\infty} \leq r$ and such that $\left|h_{n}\right| \rightarrow r \chi_{B}$ a.e.; then approximate each $h_{n}$ by polynomials.) Then, letting $n \rightarrow \infty$ (using Fatou's Lemma and upper semicontinuity), we obtain

$$
\int_{0}^{2 \pi} \int_{0}^{2 \pi} F\left(f\left(e^{i \theta}\right)+r e^{i \phi} \chi_{B}\left(e^{i \theta}\right)\right) \frac{d \phi}{2 \pi} \frac{d \theta}{2 \pi} \geq 0 .
$$


Let $B=\left\{\theta: F_{1}\left(f\left(e^{i \theta}\right)\right)<F\left(f\left(e^{i \theta}\right)\right)\right\}$. Then the above inequality gives

$$
\int_{0}^{2 \pi} F_{1} \circ f\left(e^{i \theta}\right) \frac{d \theta}{2 \pi} \geq 0
$$

This proves our first claim.

Now let $\left(r_{n}\right)$ be a sequence of positive rationals in which each positive rational is repeated infinitely many times. Define $F_{0}=F$ and then

$$
F_{n}(z)=\min \left(F_{n-1}(z), \int_{0}^{2 \pi} F_{n-1}\left(z+r_{n} e^{i \theta}\right) \frac{d \theta}{2 \pi}\right) .
$$

Then each $F_{n}$ is upper semicontinuous and if $G(z)=\inf _{n} F_{n}(z)$ then $F_{n}(z) \downarrow$ $G(z) \in[-\infty, \infty)$ everywhere. By induction, using the previous claim we have, if $f(0) \in E$,

$$
\int_{0}^{2 \pi} F_{n} \circ f\left(e^{i \theta}\right) \frac{d \theta}{2 \pi} \geq 0
$$

for every $n$ and hence by Fatou's Lemma

$$
\int_{0}^{2 \pi} G \circ f\left(e^{i \theta}\right) \frac{d \theta}{2 \pi} \geq 0
$$

This implies $G(z) \geq 0$ on $E$, and in particular $G$ is not identically $-\infty$. To show it is subharmonic we note that if $r>0$ is rational,

$$
\int_{0}^{2 \pi} G\left(z+r e^{i \theta}\right) \frac{d \theta}{2 \pi}=\lim _{n: r_{n}=r} \int_{0}^{2 \pi} F_{n-1}\left(z+r e^{i \theta}\right) \frac{d \theta}{2 \pi} \geq \lim _{n: r_{n}=r} F_{n}(z)=G(z) .
$$

If $r$ is irrational we derive

$$
\int_{0}^{2 \pi} G\left(z+r e^{i \theta}\right) \frac{d \theta}{2 \pi} \geq G(z)
$$

by taking limits (again using upper semicontinuity). This concludes the proof.

REMARKS. Let us note that the construction in the proof of the theorem gives a maximal subharmonic minorant. This implies that if $F$ is $p$-homogeneous when $p>0$ (i.e. $F(t z)=t^{p} F(z)$ when $t>0$ ) then the subharmonic minorant can also be supposed to be $p$-homogeneous. (If $G(z)$ is a subharmonic minorant of $F$ then $F(z) \geq \sup _{t>0} t^{-p} G(t z)$ by $p$-homogeneity of $F$. Since the right-hand side is subharmonic it follows that $G(z)=$ $\sup _{t>0} t^{-p} G(t z)$, and hence $G$ is $p$-homogeneous provided it is a maximal subharmonic minorant.)

Note also that the same argument but using Lemma 3.1 of [Ka] gives a similar result for plurisubharmonic minorants: 
TheOREM 2.3. Let $F: \mathbb{C}^{n} \rightarrow[-\infty, \infty)$ be an upper semicontinuous function, and let $E$ be a nonempty subset of $\mathbb{C}^{n}$. In order that for every $n$-tuple $\left(f_{1}, \ldots, f_{n}\right)$ of polynomials with $\left(f_{1}(0), \ldots, f_{n}(0)\right) \in E$ we have

$$
\int_{0}^{2 \pi} F\left(f_{1}\left(e^{i \theta}\right), \ldots, f_{n}\left(e^{i \theta}\right)\right) \frac{d \theta}{2 \pi} \geq 0
$$

it is necessary and sufficient that there is a plurisubharmonic function $G$ : $\mathbb{C}^{n} \rightarrow[-\infty, \infty)$ with $G \leq F$ and $G\left(z_{1}, \ldots, z_{n}\right) \geq 0$ for $\left(z_{1}, \ldots, z_{n}\right) \in E$.

Proof. We sketch the details of the proof that (2.4) shows the existence of a plurisubharmonic minorant. In this case we let $\Gamma$ be a dense countable subset of $\mathbb{C}^{n}$ and then let $\left(\left(w_{1}^{(m)}, w_{2}^{(m)}, \ldots, w_{n}^{(m)}\right), r_{m}\right)_{m \in \mathbb{N}}$ be a sequence in $\Gamma \times \mathbb{Q}_{+}$such that each pair $\left(\left(w_{1}, \ldots, w_{n}\right), r\right)$ with $\left(w_{1}, \ldots, w_{n}\right) \in \Gamma$ and $r \in \mathbb{Q}_{+}$is repeated infinitely often. Define $F_{0}=F$ and then inductively

$$
F_{m}(z)=\min \left(F_{m-1}(z), \int_{0}^{2 \pi} F_{m-1}\left(z_{1}+r_{m} e^{i \theta} w_{1}^{(m)}, \ldots, z_{n}+r_{n} e^{i \theta} w_{n}^{(m)}\right) \frac{d \theta}{2 \pi}\right) .
$$

The same argument now shows that $\lim _{m \rightarrow \infty} F_{m}(z)=G(z)$ is plurisubharmonic and satisfies $G\left(z_{1}, \ldots, z_{n}\right) \geq 0$ for $\left(z_{1}, \ldots, z_{n}\right) \in E$; however one must use Lemma 3.1 of $[\mathrm{Ka}]$ in place of Lemma 2.2.

Remark. After the initial preparation of the paper, we learned that a similar result was obtained by Poletsky $[\mathrm{Po}]$ by a somewhat different method.

3. Existence of a subharmonic minorant. In this section we study conditions under which a continuous $p$-homogeneous function admits a subharmonic minorant. This is greatly assisted by the theory of trigonometrically convex functions, which we develop first.

Suppose $I \subset \mathbb{R}$ is an open interval. We shall say that a function $f: I \rightarrow \mathbb{R}$ is trigonometrically convex if whenever $x<t<y$ and $y-x<\pi$ then

$$
f(t) \leq \frac{f(x) \sin (y-t)+f(y) \sin (t-x)}{\sin (y-x)} .
$$

We say $f$ is trigonometrically concave if $-f$ is trigonometrically convex. It is easily seen that a function is both trigonometrically convex and trigonometrically concave if and only if it is of the form $f(x)=A \cos x+B \sin x$; we will refer to functions of this type as sinusoidal functions. Note also that if $f(x)$ is trigonometrically convex then so is $f(-x)$.

The theory of trigonometrically convex functions is developed in [L1], [L2]. We here develop what we need, although we suspect much of it is already well known to experts. 
Lemma 3.1. Suppose $f$ is trigonometrically convex on I. Then for every $x \in I$ the left and right derivatives

$$
f_{+}^{\prime}(x)=\lim _{y \rightarrow x+} \frac{f(y)-f(x)}{y-x}, \quad f_{-}^{\prime}(x)=\lim _{y \rightarrow x-} \frac{f(y)-f(x)}{y-x}
$$

exist and satisfy

$$
-\infty<f_{-}^{\prime}(x) \leq f_{+}^{\prime}(x)<\infty .
$$

Furthermore if $|x-y| \leq \pi$ then

$$
f(y) \geq f(x) \cos (y-x)+a \sin (y-x)
$$

whenever $f_{-}^{\prime}(x) \leq a \leq f_{+}^{\prime}(x)$.

Proof. Suppose $x<t<y$ where $x, y \in I$ and $y-x<\pi$. Then

$$
\frac{f(t)-f(x)}{2 \sin \left(\frac{1}{2}(t-x)\right)} \leq \frac{f(y) \cos \left(\frac{1}{2}(t-x)\right)-f(x) \cos \left(y-\frac{1}{2}(t+x)\right)}{\sin (y-x)}
$$

by (3.1). Taking limits as $t \rightarrow x^{+}$we have

$$
\limsup _{t \rightarrow x+} \frac{f(t)-f(x)}{t-x} \leq \frac{f(y)-f(x) \cos (y-x)}{\sin (y-x)} .
$$

Taking limits as $y \rightarrow x+$ we see that

$$
\limsup _{t \rightarrow x+} \frac{f(t)-f(x)}{t-x} \leq \liminf _{y \rightarrow x+} \frac{f(y)-f(x)}{y-x}<\infty .
$$

This shows that $f_{+}^{\prime}(x)$ exists and $f_{+}^{\prime}(x)<\infty$. We further deduce that if $y>x$ with $y-x<\pi$ then

$$
f(y) \geq f(x) \cos (y-x)+f_{+}^{\prime}(x) \sin (y-x) .
$$

We note then by symmetry that $f_{-}^{\prime}(x)$ exists, $f_{-}^{\prime}(x)>-\infty$ and if $y<x$ with $x-y<\pi$ then

$$
f(y) \geq f(x) \cos (y-x)+f_{-}^{\prime}(x) \sin (y-x) .
$$

To complete the proof we need only show that $f_{-}^{\prime}(x) \leq f_{+}^{\prime}(x)$. However if $0<h<\frac{\pi}{2}$ and $x \pm h \in I$ then

$$
f(x) \leq \frac{f(x+h)+f(x-h)}{2 \cos h}
$$

so that $f_{-}^{\prime}(x) \leq f_{+}^{\prime}(x)$.

Notice that the argument yields the following:

Lemma 3.2. Suppose $f$ is continuous on $[\alpha, \beta]$ and trigonometrically convex on $(\alpha, \beta)$. Then $f_{+}^{\prime}(\alpha)$ exists, $f_{+}^{\prime}(\alpha)<\infty$ and $f(x) \geq f(\alpha) \cos (x-\alpha)$ $+f_{+}^{\prime}(\alpha) \sin (x-\alpha)$ for $x \leq \alpha+\pi$.

The following is given as a problem in [L2]. 
Proposition 3.3 [L2, p. 56]. Suppose $f:(\alpha, \beta) \rightarrow \mathbb{R}$ is a continuous function and $p>0$ is such that $\beta-\alpha<2 p \pi$. Define $F(z)$ for $z=r e^{i \theta}$ where $\alpha<p \theta<\beta$ by $F(z)=r^{p} f(p \theta)$. Then $F$ is subharmonic if and only if $f$ is trigonometrically convex.

Proof. Suppose $F$ is subharmonic. If $\alpha<s<t<\beta$ with $t-s<\pi$ then let

$$
h(x)=\frac{f(s) \sin (t-x)+f(t) \sin (x-s)}{\sin (t-s)}
$$

and then note that $F\left(r e^{i \theta}\right)-r^{p} h(p \theta)$ is subharmonic and vanishes on the rays $\theta=s / p$ and $\theta=t / p$. By the Phragmén-Lindelöf principle $F\left(r e^{i \theta}\right)-$ $r^{p} h(p \theta) \leq 0$ if $s \leq p \theta \leq t$.

Conversely assume $f$ is trigonometrically convex and that $\alpha<p \theta_{0}<\beta$. Let $z_{0}=r_{0} e^{i \theta_{0}}$. Then let $a=f_{+}^{\prime}\left(p \theta_{0}\right)$. We have, for $\left|\theta-\theta_{0}\right|$ small enough,

$$
f\left(p \theta_{0}\right) \cos p\left(\theta-\theta_{0}\right)+a \sin p\left(\theta-\theta_{0}\right) \leq f(p \theta) .
$$

Now define

$$
H\left(r e^{i \theta}\right)=r^{p}\left(f\left(p \theta_{0}\right) \cos p\left(\theta-\theta_{0}\right)+a \sin p\left(\theta-\theta_{0}\right)\right)
$$

for $\alpha<p \theta<\beta$. Then $H$ is harmonic and $H\left(z_{0}\right)=F\left(z_{0}\right)$. Now, if $\varrho$ is small enough,

$$
F\left(z_{0}\right)=H\left(z_{0}\right)=\int_{0}^{2 \pi} H\left(z_{0}+\varrho e^{i \theta}\right) \frac{d \theta}{2 \pi} \leq \int_{0}^{2 \pi} F\left(z_{0}+\varrho e^{i \theta}\right) \frac{d \theta}{2 \pi}
$$

whence $F$ is subharmonic.

Proposition 3.4. (1) If $I \subset \mathbb{R}$ is an open interval and $x, x+\pi \in I$, then $f(x)+f(x+\pi) \geq 0$ if $f$ is trigonometrically convex on $I$, and $f(x)+$ $f(x+\pi) \leq 0$ if $f$ is trigonometrically concave on $I$.

(2) Suppose $f$ is continuous on $[0, \pi]$ and satisfies $f(0)+f(\pi)=0$. Then if $f$ is trigonometrically convex or trigonometrically concave on $(0, \pi)$ it follows that $f$ is a sinusoidal function.

(3) Suppose $f$ is continuous on $[0, \pi]$ and satisfies $f(0)+f(\pi)=0$. If there exists $0<\sigma<\pi$ such that $f$ is trigonometrically convex on $(0, \sigma)$ and trigonometrically concave on $(\sigma, \pi)$ then $f_{+}^{\prime}(0)+f_{-}^{\prime}(\pi) \leq 0$. If $f_{+}^{\prime}(0)+$ $f_{-}^{\prime}(\pi)=0$ then $f$ is a sinusoidal function.

Proof. (1) It is enough to consider trigonometrically convex $f$. Then by (3.2) with $y=x+\pi$ it follows that $f(x+\pi) \geq-f(x)$.

(2) Suppose $f$ is trigonometrically convex on $(0, \pi)$ and continuous on $[0, \pi]$. By Lemma 3.2, $f_{+}^{\prime}(0)$ exists and $f(t) \geq f(0) \cos t+f_{+}^{\prime}(0) \sin t$ for $0<t<\pi$. On the other hand, letting $y \rightarrow \pi^{-}$in (3.1), one gets, for 
$0<x<t<\pi$

$$
f(t) \leq \frac{f(x)+f(\pi) \cos x}{\sin x} \sin t-f(\pi) \cos t .
$$

If $f(0)+f(\pi)=0$, then letting $x \rightarrow 0^{+}$in the preceding inequality, one obtains the upper estimate $f(t) \leq f(0) \cos t+f_{+}^{\prime}(0) \sin t$. Thus $f(t)$ is a sinusoidal function on $(0, \pi)$.

(3) We observe that

$$
f(0) \cos \sigma+f_{+}^{\prime}(0) \sin \sigma \leq f(\sigma) \leq-f(\pi) \cos \sigma-f_{-}^{\prime}(\pi) \sin \sigma .
$$

In the case $f_{+}^{\prime}(0)+f_{-}^{\prime}(\pi)=0$ we note that $f(\sigma)=f(0) \cos \sigma+f_{+}^{\prime}(0) \sin \sigma$. It follows from (3.1) that for $0<x<\sigma$ we have

$$
f(x) \leq f(0) \cos x+f_{+}^{\prime}(0) \sin x .
$$

In view of Lemma 3.2 this implies $f(x)=f(0) \cos x+f_{+}^{\prime}(0) \sin x$ for $0<$ $x<\sigma$ and a similar argument applies to $\sigma<x<\pi$.

In the next theorem a sector is a subset of the complex plane of the form $S=\left\{r e^{i \theta}: r>0, \alpha<\theta<\beta\right\}$ where the angular opening of $S$, ang $S=\beta-\alpha$, satisfies $0<\operatorname{ang} S<2 \pi$. The complementary sector $S^{\prime}$ is the interior of $\mathbb{C} \backslash S$. We call a function $F: \mathbb{C} \rightarrow \mathbb{R}$ p-homogeneous $(p>0)$ if $F(a z)=a^{p} F(z)$ for $a>0$.

TheOrem 3.5. Suppose $p>1 / 2, p \neq 1$, and $F$ is a p-homogeneous continuous function on $\mathbb{C}$. Suppose there is a sector $S$ so that $F$ is subharmonic on $S$ and superharmonic on the complementary sector $S^{\prime}$. Suppose further there is no nontrivial sector on which $F$ is harmonic. Suppose that $F(z)+F\left(e^{i \pi / p} z\right) \geq 0$ for all $z$, and there exists $z_{0} \neq 0$ so that $F\left(z_{0}\right)+F\left(e^{i \pi / p} z_{0}\right)=0$. Then there is a continuous $p$-homogeneous subharmonic function $G$ with $G(z) \leq F(z)$ for all $z \in \mathbb{C}$.

REMARK. It will be clear from the proof that ang $S^{\prime}<\frac{\pi}{p}$.

Proof. We can suppose that there is $z_{0}$ with $\left|z_{0}\right|=1$ and $F\left(z_{0}\right)+$ $F\left(e^{i \pi / p} z_{0}\right)=0$. Let $z_{0}=e^{i t_{0}}$ and $z_{1}=e^{i\left(t_{0}+\pi / p\right)}$.

We may write $F\left(r e^{i t}\right)=r^{p} f(p t)$ where $f$ is a $2 p \pi$-periodic function on $\mathbb{R}$. By Proposition 3.3, $f$ is then trigonometrically convex on any interval $I$ so that $e^{i x / p} \in S$ for $x \in I$ and trigonometrically concave on any interval $I$ so that $e^{i x / p} \in S^{\prime}$ for $x \in I$.

We begin by arguing that it is impossible that both $z_{0}, z_{1}$ belong to $S^{\prime}$. Indeed, if so then $F(z)+F\left(e^{i \pi / p} z\right)$ is superharmonic on a nontrivial sector containing $z_{0}$. Equivalently $g(x)=f(x)+f(x+\pi) \geq 0$ is trigonometrically concave on an interval $\left(p t_{0}-\delta, p t_{0}+\delta\right)$ where $\delta>0$. Since $g(t)$ has a minimum at $x=p t_{0}$, for $|\tau|<\delta$ we have

$$
0=2(\cos \tau) g\left(p t_{0}\right) \geq g\left(p t_{0}-\tau\right)+g\left(p t_{0}+\tau\right)
$$


so that $g \equiv 0$ on $\left(p t_{0}-\delta, p t_{0}+\delta\right)$. Since both $f(x)$ and $f(x+\pi)$ are trigonometrically concave in this interval and $f(x)+f(x+\pi) \equiv 0$, we conclude that both are also trigonometrically convex, and hence sinusoidal by Proposition 3.4(2). This contradicts our assumptions on $F$ (which becomes harmonic on some sector).

Next we argue that the arc $A=\left\{e^{i t}: t_{0}<t<t_{0}+\frac{\pi}{p}\right\}$ cannot be contained in either $S$ or $S^{\prime}$. This follows immediately from Proposition 3.4(2) since $f$ is then either trigonometrically convex or trigonometrically concave on $\left(p t_{0}, p t_{0}+\pi\right)$.

Note that since $g$ has a minimum at $p t_{0}$ we have

$$
\begin{aligned}
& f_{-}^{\prime}\left(p t_{0}\right)+f_{-}^{\prime}\left(p t_{0}+\pi\right) \leq 0, \\
& f_{+}^{\prime}\left(p t_{0}\right)+f_{+}^{\prime}\left(p t_{0}+\pi\right) \geq 0 .
\end{aligned}
$$

We will next argue that it is impossible for either $z_{0}$ or $z_{1}$ to fall in the sector $S^{\prime}$. Let us suppose $z_{1} \in S^{\prime}$; the other case is similar and may be reduced to this case by considering $F(\bar{z})$. Then $z_{0}$ cannot be in $S^{\prime}$ and so $\alpha \leq t_{0}<\beta<t_{0}+\frac{\pi}{p}<\alpha+2 \pi$. Now by Proposition 3.4(3) we have

$$
f_{+}^{\prime}\left(p t_{0}\right)+f_{-}^{\prime}\left(p t_{0}+\pi\right) \leq 0,
$$

and the fact that $z_{1} \in S^{\prime}$ entails that $f$ is trigonometrically concave in a neighborhood of $p t_{0}+\pi$, so $f_{-}^{\prime}\left(p t_{0}+\pi\right) \geq f_{+}^{\prime}\left(p t_{0}+\pi\right)$ by Lemma 3.1. Combined with (3.4) this yields

$$
f_{+}^{\prime}\left(p t_{0}\right)+f_{-}^{\prime}\left(p t_{0}+\pi\right)=0 .
$$

Hence by Proposition $3.4(3), f$ is sinusoidal on $\left(p t_{0}, p t_{0}+\pi\right)$ contrary to assumption.

Thus neither $z_{0}$ nor $z_{1}$ belongs to $S^{\prime}$, and $A$ is not contained in one sector. So at least one of the two points is contained inside $S$. Again we can suppose $z_{0} \in S$. Hence we can suppose $\alpha<t_{0}<\beta<\alpha+2 \pi \leq t_{0}+\frac{\pi}{p}$.

Now let

$$
h(x)=f\left(p t_{0}\right) \cos \left(x-p t_{0}\right)+f_{+}^{\prime}\left(p t_{0}\right) \sin \left(x-p t_{0}\right) .
$$

Then by Lemma 3.1 we have $h(x) \leq f(x)$ on a neighborhood of $p t_{0}$. Also since by Proposition 3.4(3), $f_{+}^{\prime}\left(p t_{0}\right)+f_{-}^{\prime}\left(p t_{0}+\pi\right) \leq 0$ and $f$ is trigonometrically convex on $\left(p t_{0}+\pi, p t_{0}+\pi+\delta\right)$ for some $\delta>0$ we have $h(x) \leq f(x)$ for $p t_{0}+\pi \leq x<p t_{0}+\pi+\delta$.

Now note that by Lemma 3.2 (or by the Phragmén-Lindelöf theorem) $h(x) \leq f(x)$ for $p t_{0} \leq x \leq p \beta$ and for $p \alpha+2 p \pi \leq x \leq p t_{0}+\pi$. Since $f(x)-h(x)$ is trigonometrically concave for $p \beta<x<p \alpha+2 p \pi$, and is positive in the adjacent intervals, it cannot change sign on $(p \beta, p \alpha+2 p \pi)$, again by the Phragmén-Lindelöf theorem. This means $h(x) \leq f(x)$ for all $x$ in a neighborhood of $\left[p t_{0}, p t_{0}+\pi\right]$. 
Let $T=\left\{r e^{i \theta}: r>0, t_{0}<\theta<t_{0}+\frac{\pi}{p}\right\}$ and define $H\left(r e^{i \theta}\right)=r^{p} h(p \theta)$ for $t_{0}<\theta<t_{0}+\frac{\pi}{p}$. Note that $T$ contains the sector $S^{\prime}$. Let

$$
G(z)= \begin{cases}H(z) & \text { if } z \in T \\ F(z) & \text { if } z \notin T .\end{cases}
$$

Clearly $G(z) \leq F(z)$ for all $z \in \mathbb{C}$, and $G$ is subharmonic on both $T$ and its complementary sector $T^{\prime}$. Since $h(x) \leq f(x)$ in a neighborhood of both $p t_{0}$ and $p t_{0}+\pi$ it is easy to see that $G$ is then subharmonic on $\mathbb{C} \backslash 0$. Finally this implies that $G(z)+G\left(e^{i \pi / p} z\right) \geq 0$ ([L2]) for all $z$ and so by integrating

$$
\int_{0}^{2 \pi} G\left(r e^{i \theta}\right) \frac{d \theta}{2 \pi} \geq 0, \quad r>0 .
$$

Hence $G$ is subharmonic on $\mathbb{C}$.

Corollary 3.6. (1) Under the conditions of Theorem 3.5 there is a unique $z_{0}$ with $\left|z_{0}\right|=1$ and $F\left(z_{0}\right)+F\left(e^{i \pi / p} z_{0}\right)=0$.

(2) If , in addition, $F(z)=F(\bar{z})$ for all $z \in \mathbb{C}$ then $z_{0}=e^{-i \pi /(2 p)}$ or $z_{0}=-e^{-i \pi /(2 p)}$.

Proof. From Proposition 3.4 (or the Phragmén-Lindelöf principle) it is clear that if $F\left(z_{0}\right)+F\left(e^{i \pi / p} z_{0}\right)=0$ then any subharmonic minorant $G$ is harmonic on the sector bounded by the rays through $z_{0}$ and $e^{i \pi / p} z_{0}$. The minorant $G$ constructed in the proof of Theorem 3.5 is harmonic on exactly one sector of angle $\frac{\pi}{p}$. This proves (1). For (2) we observe that the uniqueness of $z_{0}$ implies that $\bar{z}_{0}=e^{i \pi / p} z_{0}$.

We shall use the following version of this theorem:

Theorem 3.7. Suppose $p>1, p \neq 2$, and $F$ is a symmetric $p$-homogeneous continuous function on $\mathbb{C}$. Suppose there are sectors $S, T$ with $\operatorname{ang} S+\operatorname{ang} T=\pi$ so that $F$ is subharmonic on $S \cup(-S)$ and superharmonic on $T \cup(-T)$. Suppose further there is no nontrivial sector on which $F$ is harmonic. Suppose that $F(z)+F\left(e^{i \pi / p} z\right) \geq 0$ for all $z$, and there exists $z_{0} \neq 0$ so that $F\left(z_{0}\right)+F\left(e^{i \pi / p} z_{0}\right)=0$. Then there is a continuous $p$-homogeneous subharmonic function $G$ with $G(z) \leq F(z)$ for all $z \in \mathbb{C}$.

Proof. Define $\widetilde{F}(z)=F\left(z^{1 / 2}\right)$ (this definition is unambiguous). Then $\widetilde{F}$ is $p / 2$-homogeneous and satisfies the hypotheses of Theorem 3.5. Hence there is a $p / 2$-homogeneous subharmonic function $\widetilde{G}$ with $\widetilde{G}(z) \leq \widetilde{F}(z)$. Define $G(z)=\widetilde{G}\left(z^{2}\right)$ and the theorem follows.

Corollary 3.8. Suppose, under the conditions of Theorem 3.7, we also have $F(z)=F(\bar{z})$ for $z \in \mathbb{C}$. Then either

$$
F\left(e^{-i \pi /(2 p)}\right)+F\left(e^{i \pi /(2 p)}\right)=0,
$$


or

$$
F\left(e^{i(\pi / 2-\pi /(2 p))}\right)+F\left(e^{i(\pi / 2+\pi /(2 p))}\right)=0 .
$$

Proof. This follows immediately from Corollary 3.6.

4. The norm of $a I+b H$. Let us denote the identity operator by $I$, the Hilbert transform on the unit circle $\mathbb{T}$ by $H=H^{\mathbb{T}}$, and define

$$
B_{p}=\max _{x \in \mathbb{R}} \frac{|a x-b+(b x+a) \tan \gamma|^{p}+|a x-b-(b x+a) \tan \gamma|^{p}}{|x+\tan \gamma|^{p}+|x-\tan \gamma|^{p}}
$$

where $\gamma=\frac{\pi}{2 p}$.

Note that this is consistent with the formula (see (1.10))

$$
B_{p}=\left\|a I+b H^{\mathbb{R}}\right\|_{L^{p}(\mathbb{R})}^{p}=\|a I+b \mathfrak{h}\|_{l_{2}^{p}}^{p}
$$

discussed in the Introduction since $B_{p}$ can be defined equivalently by

$$
B_{p}=\left(a^{2}+b^{2}\right)^{p / 2} \max _{0 \leq \theta \leq 2 \pi} \frac{\left|\cos \left(\theta+\theta_{0}\right)\right|^{p}+\left|\cos \left(\theta+\theta_{0}+\frac{\pi}{p}\right)\right|^{p}}{|\cos \theta|^{p}+\left|\cos \left(\theta+\frac{\pi}{p}\right)\right|^{p}},
$$

where $\tan \theta_{0}=b / a$. This can be seen by letting $x=\cot \left(\theta+\frac{\pi}{2 p}\right)$.

TheOREM 4.1. Let $f \in L^{p}(\mathbb{T})$ for $1<p<\infty$ be a real-valued function. Then for any $a, b \in \mathbb{R}$,

$$
\left\|\left(a I+b H^{\mathbb{T}}\right) f\right\|_{L^{p}(\mathbb{T})}^{p} \leq B_{p}\|f\|_{L^{p}(\mathbb{T})}^{p}
$$

where the constant, $B_{p}$, is sharp. In other words, $\left\|a I+b H^{\mathbb{T}}\right\|_{L^{p}(\mathbb{T})}=B_{p}^{1 / p}$.

It is well known that for real $a, b$ the operator norms of $a I+b H^{\mathbb{T}}$ in the real and complex $L^{p}$ spaces coincide.

Proof. If $p=2$ then clearly

$$
\left\|a I+b H^{\mathbb{T}}\right\|_{L^{2}(\mathbb{T})}^{2}=a^{2}+b^{2}=B_{2},
$$

and so we can assume $p \neq 2$. We proceed with the following lemma.

Lemma 4.2. Let $B_{p}$ be given by (4.1). Then there exists a subharmonic function $G(z)$ such that

$$
|a \operatorname{Re} z+b \operatorname{Im} z|^{p} \leq B_{p}|\operatorname{Re} z|^{p}-G(z)
$$

Proof. Since the case $b=0$ is trivial we assume $b \neq 0$. Clearly, letting $\Phi(z)=B_{p}|\operatorname{Re} z|^{p}-|a \operatorname{Re} z+b \operatorname{Im} z|^{p}$ gives a function of the form $\Phi\left(r e^{i t}\right)=$ $r^{p} \phi(t)$, where $\phi(t)=B_{p}|\cos t|^{p}-|a \cos t+b \sin t|^{p}$. Note that $\phi(t)$ is $\pi$-periodic and continuously differentiable. One can readily see that (4.2) implies

$$
\min _{0 \leq t \leq 2 \pi}[\phi(t)+\phi(t+\pi / p)]=0 .
$$


To determine where $\Phi(z)$ is subharmonic or superharmonic, we observe that $\Delta \Phi \geq 0$ is equivalent to

$$
B_{p}|\operatorname{Re} z|^{p-2} \geq\left(a^{2}+b^{2}\right)|a \operatorname{Re} z+b \operatorname{Im} z|^{p-2} .
$$

So in order for $\Phi(z)$ to be subharmonic, the following must be true:

$$
|a+b \tan t|^{p-2} \leq \frac{B_{p}}{a^{2}+b^{2}}
$$

Therefore we see that for $p \neq 2$ there will indeed be exactly two separate "double sectors" where $\Phi(z)$ is subharmonic, and superharmonic in their complement, and we can use Theorem 3.7 to conclude that $\Phi$ has a subharmonic minorant. Thus the lemma is proved.

We also need the following observation.

LEMma 4.3. Let $G(z)$ be the subharmonic minorant found in the proof of Lemma 4.2. Then $G(x) \geq 0$ for all $x \in \mathbb{R}$.

Proof. Let $\Phi(z)=B_{p}|\operatorname{Re} z|^{p}-|a \operatorname{Re} z+b \operatorname{Im} z|^{p}$, and let $G(z)$ be the subharmonic minorant. As in the proof of Lemma 4.2 we may assume $b \neq 0$.

Notice that $\Phi(x) \geq 0$ for real $x$. This follows from the definition of $B_{p}$; in particular, from (4.1) where $x=0$ we have

$$
B_{p} \geq \frac{|a \tan \gamma-b|^{p}+|a \tan \gamma+b|^{p}}{2|\tan \gamma|^{p}}=\frac{|a|^{p}}{2}\left(|1-c|^{p}+|1+c|^{p}\right),
$$

where $c=\frac{b}{a \tan \gamma}$. By the convexity of $x^{p}$ it follows that

$$
|1+c|^{p}+|1-c|^{p} \geq 2
$$

for $p \geq 1$. Thus, we can conclude that $B_{p} \geq|a|^{p}$, and hence

$$
\Phi(x)=B_{p}|x|^{p}-|a x|^{p} \geq 0
$$

for all $x \in \mathbb{R}$.

To show that $G(x) \geq 0$ for real $x$, suppose first $p>2$. Then we argue that $G(x)=\Phi(x)$ if $x \in \mathbb{R}$. To prove this statement, we set $S=\{z$ : $G(z) \neq \Phi(z)\}$. Observe from the construction of the minorant in the proof of Theorem 3.7 that $S$ is contained in a double sector which has an angle of opening $\frac{\pi}{p}$. It is clear from (4.7) that the imaginary axis is contained in a double sector where $\Phi(z)(z \neq 0)$ is superharmonic, and hence it lies in $S$. If $S$ is to contain the real axis as well, then its angle of opening must be at least $\frac{\pi}{2}$. Since $p>2$, we have a contradiction.

Now suppose $1<p<2$, and hence $\frac{\pi}{2}<\frac{\pi}{p}<\pi$. Notice that on the imaginary axis

$$
G(i x) \leq \Phi(i x)=-|b x|^{p}<0, \quad x \neq 0,
$$


provided $b \neq 0$. If $G(x) \leq 0$ for some $x \neq 0$ on the real axis as well then by the Phragmén-Lindelöf theorem (or simply by (3.1) and Lemma 3.1) $G(z)<0$ either in the right or left half-plane. This contradicts the inequality $G(z)+G\left(z e^{i \pi / p}\right) \geq 0$ which holds for every $z$ since $G(z)$ is subharmonic and $p$-homogeneous in $\mathbb{C}$.

Our next step is to use Lemma 4.2 and replace $z$ with $h(z)=u(z)+i v(z)$, where $h(z)$ is analytic in the unit disc $\mathbb{D}$. Here $u(z)$ and $v(z)$ are the harmonic extensions to $\mathbb{D}$ of the functions $f$ and $\widetilde{f}=H^{\mathbb{T}} f$ respectively, so that $v(0)=0$, and $h=f+i \tilde{f} \in H^{p}(\mathbb{T})$. Then (4.4) becomes

$$
|a u(z)+b v(z)|^{p} \leq B_{p}|u(z)|^{p}-G(h(z)),
$$

where $z \in \mathbb{D}$. Note that since $h$ is analytic, $G \circ h$ is still subharmonic in $\mathbb{D}$. Because $v(0)=0$, we can apply Lemma 4.3 and observe that $G(h(0)) \geq 0$. So we can let $z=r e^{i \theta}, 0<r<1$, and integrate both sides of (4.8) over $0 \leq \theta \leq 2 \pi$. Using the sub-mean-value property for $G \circ h$, and letting $r \rightarrow 1$, we conclude that

$$
\|a f+b \widetilde{f}\|_{L^{p}(\mathbb{T})}^{p} \leq B_{p}\|f\|_{L^{p}(\mathbb{T})}^{p},
$$

which proves (4.3).

To see that the constant, $B_{p}$, is sharp, we need to show

$$
\left\|a I+b H^{\mathbb{T}}\right\|_{L^{p}(\mathbb{T})}^{p} \geq B_{p} .
$$

Recall the function used in $[\mathrm{Pi}]$,

$$
g_{\gamma}(z)=\left(\frac{1+z}{1-z}\right)^{2 \gamma / \pi}, \quad g_{\gamma}(0)=1,
$$

where $\gamma<\frac{\pi}{2 p}$ and $1<p<2$. It has the property that

$$
\operatorname{Im} g_{\gamma}\left(e^{i \theta}\right)= \begin{cases}\tan \gamma \operatorname{Re} g_{\gamma}\left(e^{i \theta}\right) & \text { if } 0<\theta<\pi, \\ -\tan \gamma \operatorname{Re} g_{\gamma}\left(e^{i \theta}\right) & \text { if }-\pi<\theta<0 .\end{cases}
$$

So consider a function of the form $f_{\gamma}=\alpha \operatorname{Re} g_{\gamma}+\beta \operatorname{Im} g_{\gamma}$, where $\alpha, \beta \in \mathbb{R}$. Notice that $\tilde{f}_{\gamma}=H^{\mathbb{T}} f_{\gamma}=\alpha \operatorname{Im} g_{\gamma}-\beta \operatorname{Re} g_{\gamma}+\beta$ and so for fixed $\alpha, \beta$ we have

$$
\begin{aligned}
& \left\|a I+b H^{\mathbb{T}}\right\|_{L^{p}(\mathbb{T})} \geq \frac{\left\|a f_{\gamma}+b \widetilde{f}_{\gamma}\right\|_{L^{p}(\mathbb{T})}}{\left\|f_{\gamma}\right\|_{L^{p}(\mathbb{T})}} \\
& \quad \geq\left(\frac{\int_{\mathbb{T}}\left|(a \alpha-b \beta) \operatorname{Re} g_{\gamma}\left(e^{i \theta}\right)+(a \beta+b \alpha) \operatorname{Im} g_{\gamma}\left(e^{i \theta}\right)\right|^{p} \frac{d \theta}{2 \pi}}{\int_{\mathbb{T}}\left|a \operatorname{Re} g_{\gamma}\left(e^{i \theta}\right)+b \operatorname{Im} g_{\gamma}\left(e^{i \theta}\right)\right|^{p} \frac{d \theta}{2 \pi}}\right)^{1 / p}-\frac{|b \beta|}{\left\|f_{\gamma}\right\|_{L^{p}(\mathbb{T})}} .
\end{aligned}
$$


We can use (4.11) and the fact that $\operatorname{Re} g_{\gamma}\left(e^{i \theta}\right)=\cos \gamma\left|\cot \frac{\theta}{2}\right|^{2 \gamma / \pi}$ is an even function to rewrite the right-hand side of the above as

$$
\begin{array}{r}
\left(\frac{|a \alpha-b \beta+(a \beta+b \alpha) \tan \gamma|^{p}+|a \alpha-b \beta-(a \beta+b \alpha) \tan \gamma|^{p}}{|\alpha+\beta \tan \gamma|^{p}+|\alpha-\beta \tan \gamma|^{p}}\right)^{1 / p} \\
-\frac{|b \beta|}{\left\|f_{\gamma}\right\|_{L^{p}(\mathbb{T})}} .
\end{array}
$$

If we let $\gamma \rightarrow \frac{\pi}{2 p}$ then the second term in the preceding expression tends to zero. Thus,

$$
\begin{aligned}
& \left\|a I+b H^{\mathbb{T}}\right\|_{L^{p}(\mathbb{T})} \\
& \geq \max _{\alpha, \beta \in \mathbb{R}}\left(\frac{\left|a \alpha-b \beta+(a \beta+b \alpha) \tan \gamma^{\prime}\right|^{p}+\left|a \alpha-b \beta-(a \beta+b \alpha) \tan \gamma^{\prime}\right|^{p}}{\left|\alpha+\beta \tan \gamma^{\prime}\right|^{p}+\left|\alpha-\beta \tan \gamma^{\prime}\right|^{p}}\right)^{1 / p},
\end{aligned}
$$

where $\gamma^{\prime}=\frac{\pi}{2 p}$.

Letting $x=\alpha / \beta$, we see that (4.10) holds and therefore the constant is sharp for $1<p<2$. For $2<p<\infty$, one can employ a similar argument by instead using the function $g_{\gamma}(z)=i\left(\frac{1+z}{1-z}\right)^{2 \gamma / \pi}$.

We also have the following corollary.

Corollary 4.4. Let $f \in L^{p}(\mathbb{R})$ for $1<p<\infty$ be a real-valued function. Then for any $a, b \in \mathbb{R}$,

$$
\left\|\left(a I+b H^{\mathbb{R}}\right) f\right\|_{L^{p}(\mathbb{R})}^{p} \leq B_{p}\|f\|_{L^{p}(\mathbb{R})}^{p},
$$

where the sharp constant, $B_{p}$, is given by (4.1). Equivalently, the operator norm is given by $\left\|a I+b H^{\mathbb{R}}\right\|_{L^{p}(\mathbb{R})}=B_{p}^{1 / p}$.

Proof. Inequality (4.12) can be shown by using a standard argument known as "blowing up the circle". This idea, due to A. Zygmund [Z, Chapter XVI, Theorem 3.8], involves a reduction to the periodic case, followed by an application of Theorem 4.1. For examples of this argument, see Theorem 3.1 in $[\mathrm{HV}]$ or Proposition 3.5 in $[\mathrm{BG}]$.

We can show $B_{p}$ is sharp by reduction to the case of the circle using transference. For instance, Theorem 3.8 in $[\mathrm{BG}]$ where $\mu_{1}=a-i b, \mu_{2}=$ $a+i b$, and $\mu=a$, combined with our Theorem 4.1, yields

$$
\left\|a I+b H^{\mathbb{R}}\right\|_{L^{p}(\mathbb{R})} \geq\left\|a I+b H^{\mathbb{T}}\right\|_{L^{p}(\mathbb{T})}=B_{p}^{1 / p}
$$

5. Related inequalities. In this section we give some other applications of Theorem 3.7, which illustrate its possible uses. Let us suppose $1<p<\infty$ and $u \in L^{p}(\mathbb{T})$; let $v=H^{\mathbb{T}} u$ and $f=u+i v$, where $u$ and $v$ are real-valued. In particular we study best constant inequalities which involve 
the $L^{p}$-norm of $\Phi_{t}(u+i v)$, where

$$
\Phi_{t}(z)=\left(x^{2}+t^{2} y^{2}\right)^{1 / 2}, \quad z=x+i y \in \mathbb{C}, t \geq 0 .
$$

We shall say $u$ has mean zero if $\int_{0}^{2 \pi} u\left(e^{i \theta}\right) d \theta=0$. Note that $v$ always has mean zero.

Theorem 5.1. Let $1<p<\infty$, and $p^{*}=\max \left(p, p^{\prime}\right)$. If $t \geq 1$ then

$$
\left\|\left(u^{2}+t^{2} v^{2}\right)^{1 / 2}\right\|_{p} \leq\left(\sin ^{2} \frac{\pi}{2 p^{*}}+t^{2} \cos ^{2} \frac{\pi}{2 p^{*}}\right)^{1 / 2}\|f\|_{p} .
$$

If $0<t \leq 1$ then

$$
\left(\sin ^{2} \frac{\pi}{2 p^{*}}+t^{2} \cos ^{2} \frac{\pi}{2 p^{*}}\right)^{1 / 2}\|f\|_{p} \leq\left\|\left(u^{2}+t^{2} v^{2}\right)^{1 / 2}\right\|_{p} .
$$

If we additionally suppose $u$ has mean zero, then if $t \geq 1$,

$$
\left(\cos ^{2} \frac{\pi}{2 p^{*}}+t^{2} \sin ^{2} \frac{\pi}{2 p^{*}}\right)^{1 / 2}\|f\|_{p} \leq\left\|\left(u^{2}+t^{2} v^{2}\right)^{1 / 2}\right\|_{p}
$$

and if $0<t<1$,

$$
\left\|\left(u^{2}+t^{2} v^{2}\right)^{1 / 2}\right\|_{p} \leq\left(\cos ^{2} \frac{\pi}{2 p^{*}}+t^{2} \sin ^{2} \frac{\pi}{2 p^{*}}\right)^{1 / 2}\|f\|_{p} .
$$

In each case the constant is sharp.

Proof. Let us first note that the given constants are sharp by a slight variation of the argument in Theorem 4.1. Define, as before,

$$
g_{\gamma}(z)=\left(\frac{1+z}{1-z}\right)^{2 \gamma / \pi}
$$

Then $g_{\gamma} \in H^{p}$ provided $2 \gamma p<\pi$. Let $f_{\gamma}=g_{\gamma}-1 \in H_{0}^{p}$ and $u_{\gamma}=\operatorname{Re} f_{\gamma}$, $v_{\gamma}=\operatorname{Im} f_{\gamma}$. Now suppose $0 \leq t_{0}, t_{1}<\infty$. If $2 \gamma p<\pi$ we deduce from (4.11) that

$$
\left|\left\|\left(u_{\gamma}^{2}+t_{j}^{2} v_{\gamma}^{2}\right)^{1 / 2}\right\|_{p}-\left(\cos ^{2} \gamma+t_{j}^{2} \sin ^{2} \gamma\right)^{1 / 2}\left\|g_{\gamma}\right\|_{p}\right| \leq 1 .
$$

Hence since $\left\|g_{\gamma}\right\|_{p} \rightarrow \infty$ as $\gamma \rightarrow \frac{\pi}{2 p}$ it follows that

$$
\lim _{\gamma \rightarrow \pi /(2 p)} \frac{\left\|\left(u_{\gamma}^{2}+t_{1}^{2} v_{\gamma}^{2}\right)^{1 / 2}\right\|_{p}}{\left\|\left(u_{\gamma}^{2}+t_{0}^{2} v_{\gamma}^{2}\right)^{1 / 2}\right\|_{p}}=\frac{\left(\cos ^{2} \frac{\pi}{2 p}+t_{1}^{2} \sin ^{2} \frac{\pi}{2 p}\right)^{1 / 2}}{\left(\cos ^{2} \frac{\pi}{2 p}+t_{0}^{2} \sin ^{2} \frac{\pi}{2 p}\right)^{1 / 2}} .
$$

This equation, combined with a similar equation derived from if $f_{\gamma}$ (thus interchanging the roles of $u_{\gamma}$ and $v_{\gamma}$ ) quickly shows that each constant in Theorem 5.1 is sharp even when $u$ has mean zero.

We now turn to the proof of (5.1)-(5.4). We will write $z=x+i y$ for $z \in \mathbb{C}$. We now suppose $1<p<\infty$ (with $p \neq 2$ ) and $0<t<\infty$ are fixed. 
Recall that $\Phi_{t}(z)=\left(x^{2}+t^{2} y^{2}\right)^{1 / 2}$ where $z=x+i y$. We set

$$
F^{+}(z)=\Phi_{t}(z)^{p}-c|z|^{p}, \quad z \in \mathbb{C},
$$

where

$$
c=\frac{1}{2} \min _{-\pi<\theta \leq \pi}\left(\Phi_{t}\left(e^{i(\theta+\pi / p)}\right)^{p}+\Phi_{t}\left(e^{i \theta}\right)^{p}\right),
$$

and

$$
F^{-}(z)=d|z|^{p}-\Phi_{t}(z)^{p}, \quad z \in \mathbb{C},
$$

where

$$
d=\frac{1}{2} \max _{-\pi \leq \theta \leq \pi}\left(\Phi_{t}\left(e^{i(\theta+\pi / p)}\right)^{p}+\Phi_{t}\left(e^{i \theta}\right)^{p}\right) .
$$

We shall show that the conditions of Theorem 3.7 are met for both $F^{+}$ and $F^{-}$. By the symmetry conditions on $F=F^{+}, F^{-}$it is only necessary to show that $\Delta F\left(e^{i \theta}\right)$ changes sign at most once in the first quadrant. Note that by construction we have

$$
\min _{|z|=1}\left(F(z)+F\left(e^{i \pi / p} z\right)\right)=0
$$

We thus calculate:

$$
\Delta \Phi_{t}(x+i y)^{p}=p\left(1+t^{2}\right) \Phi_{t}(x+i y)^{p-2}+p(p-2)\left(x^{2}+t^{4} y^{2}\right) \Phi_{t}(x+i y)^{p-4} .
$$

Hence

$$
\Delta \Phi_{t}(z)^{p}=p(p-1)\left(1+t^{2}\right) \Phi_{t}(z)^{p-2}-p(p-2) t^{2}|z|^{2} \Phi_{t}(z)^{p-4} .
$$

We next show that for any choice of $a$ it follows that $\Delta\left(\Phi_{t}^{p}-a|z|^{p}\right)$ vanishes on at most one ray in the first quadrant. In fact if $\lambda(z)=\Phi_{t}(z) /|z|$ then by (5.6), $\Delta\left(\Phi_{t}(z)^{p}-a \Phi_{1}(z)^{p}\right)=|z|^{p-2}\left(p(p-1)\left(1+t^{2}\right) \lambda^{p-2}-p(p-2) t^{2} \lambda^{p-4}-p^{2} a\right)$, and $\lambda(z)$ lies in the interval $[t, 1]$ or $[1, t]$. Now the function

$$
\varphi(\lambda)=p(p-1)\left(1+t^{2}\right) \lambda^{p-2}-p(p-2) t^{2} \lambda^{p-4}-p^{2} a
$$

vanishes at most once in this interval since $\varphi^{\prime}(\lambda)$ vanishes (for $\lambda>0$ ) only when

$$
\lambda^{2}=\frac{(p-4) t^{2}}{(p-1)\left(1+t^{2}\right)}, \quad \lambda^{2}<\min \left(1, t^{2}\right) .
$$

It follows that if $F=F^{+}$or $F=F^{-}$, then $\Delta F$ vanishes on at most one ray in the first quadrant. Since for some $z_{0} \neq 0$ we have $F\left(z_{0} e^{i \pi / p}\right)+F\left(z_{0}\right)=0$ this implies it vanishes on exactly one ray (if it is subharmonic or superharmonic then it must be harmonic on some sector of angle $\frac{\pi}{p}$ by Propositions 3.3 and 3.4(2)). Now Theorem 3.7 can be applied and $F$ admits a continuous $p$-homogeneous subharmonic minorant $G$. Furthermore an application of 
Corollary 3.8 shows that

$c=\left(\cos ^{2} \frac{\pi}{2 p^{*}}+t^{2} \sin ^{2} \frac{\pi}{2 p^{*}}\right)^{p / 2}, \quad d=\left(\sin ^{2} \frac{\pi}{2 p^{*}}+t^{2} \cos ^{2} \frac{\pi}{2 p^{*}}\right)^{p / 2}, \quad t \geq 1$,

and

$c=\left(\sin ^{2} \frac{\pi}{2 p^{*}}+t^{2} \cos ^{2} \frac{\pi}{2 p^{*}}\right)^{p / 2}, \quad d=\left(\cos ^{2} \frac{\pi}{2 p^{*}}+t^{2} \sin ^{2} \frac{\pi}{2 p^{*}}\right)^{p / 2}$,

$0<t<1$.

In either case $F=F^{+}$or $F=F^{-}$we have $G(0)=0$ by homogeneity. We check conditions for $G(x) \geq 0$ if $x \in \mathbb{R}$.

First assume that $t \geq 1$ and

$$
F(z)=F^{-}(z)=\left(\sin ^{2} \frac{\pi}{2 p^{*}}+t^{2} \cos ^{2} \frac{\pi}{2 p^{*}}\right)^{p / 2}|z|^{p}-\left(x^{2}+t^{2} y^{2}\right)^{p / 2} .
$$

We split into two cases. If $p>2$ then $G(1)=F(1)$ and so $G(1) \geq 0$.

If $p<2$ then $G$ is harmonic on the sector $-\frac{\pi}{2 p}<\arg z<\frac{\pi}{2 p}$ and of the form $G\left(r e^{i \theta}\right)=A r^{p} \cos p \theta$ by the construction of $G$ in the proof of Theorem 3.5. Next, $G\left(e^{i \theta}\right)=F\left(e^{i \theta}\right)$ for $\theta>\frac{\pi}{2 p}$ and this is nonpositive for $\frac{\pi}{2 p}<\theta<\frac{\pi}{2 p}+\delta$ for suitable $\delta>0$. Then since $G$ is $p$-homogeneous and subharmonic, $G\left(e^{i \theta}\right)+G\left(e^{i(\theta+\pi / p)}\right) \geq 0$ by Propositions 3.3 and 3.4(1), so $G\left(e^{i \theta}\right) \geq 0$ for $-\frac{\pi}{2 p}<\theta<-\frac{\pi}{2 p}+\delta$. But this implies $A>0$ and so $G(1)>0$. Thus if $t \geq 1$ we have $G(x) \geq 0$ for $1<p<\infty$.

Note that if $0<t<1$ and

$$
F(z)=F^{-}(z)=\left(\cos ^{2} \frac{\pi}{2 p^{*}}+t^{2} \sin ^{2} \frac{\pi}{2 p^{*}}\right)^{p / 2}|z|^{p}-\left(x^{2}+t^{2} y^{2}\right)^{p / 2},
$$

then $F(1)<0$ and so $G(1)<0$.

If $F=F^{+}$we similarly deduce that $G(1) \geq 0$ only when $0<t \leq 1$.

The remainder of the argument is standard. Define $f$ for $|z|<1$ by $f(z)=\sum_{n \geq 0} \widehat{f}(n) z^{n}$ where $\widehat{f}(0)$ is real. Then $G \circ f$ is subharmonic and hence

$$
0 \leq \int_{0}^{2 \pi} G\left(f\left(r e^{i \theta}\right)\right) \frac{d \theta}{2 \pi}, \quad 0<r<1
$$

Thus

$$
\int_{0}^{2 \pi} F\left(f\left(r e^{i \theta}\right)\right) \frac{d \theta}{2 \pi} \geq 0
$$

and letting $r \rightarrow 1$ we will obtain (5.1), (5.2), (5.3) or (5.4) according to the choice of $F^{+}$or $F^{-}$and $p$. 
Before giving our next application, let us note that the following theorem is an easy application of the known estimate for the Hilbert transform [Pi]:

Theorem 5.2. If $2 \leq p<\infty$ and $0<t<\infty$ then

$$
\left\|\left(u^{2}+t^{2} v^{2}\right)^{1 / 2}\right\|_{p} \leq\left(1+t^{2} \cot ^{2} \frac{\pi}{2 p}\right)^{1 / 2}\|u\|_{p} .
$$

If $1<p \leq 2$ and $0<t<\infty$ then if $u$ has mean zero,

$$
\left(1+t^{2} \cot ^{2} \frac{\pi}{2 p}\right)^{1 / 2}\|u\|_{p} \leq\left\|\left(u^{2}+t^{2} v^{2}\right)\right\|_{p}
$$

Proof. For (5.7) note that if $p / 2 \geq 1$ then

$$
\left\|\left(u^{2}+t^{2} v^{2}\right)^{1 / 2}\right\|_{p} \leq\left(\|u\|_{p}^{2}+t^{2}\|v\|_{p}^{2}\right)^{1 / 2}
$$

by Minkowski's inequality, and the estimate $\|v\|_{p} \leq \cot \frac{\pi}{2 p}\|u\|_{p}$ completes the proof. For (5.8) if $p / 2 \leq 1$ we have instead

$$
\left\|\left(u^{2}+t^{2} v^{2}\right)^{1 / 2}\right\|_{p} \geq\left(\|u\|_{p}^{2}+t^{2}\|v\|_{p}^{2}\right)^{1 / 2}
$$

and $\|v\|_{p} \geq \cot \frac{\pi}{2 p}\|u\|_{p}$ since $u$ has mean zero.

We will now consider the reverse inequalities to those of Theorem 5.2.

Theorem 5.3. Suppose either that $0<t<\infty$ and $3 \leq p<\infty$, or that $\sqrt{1-p / 3} \leq t<\infty$ and $2<p<3$. Then if $u$ has mean zero,

$$
\left(1+t^{2} \tan ^{2} \frac{\pi}{2 p}\right)^{1 / 2}\|u\|_{p} \leq\left\|\left(u^{2}+t^{2} v^{2}\right)^{1 / 2}\right\|_{p} .
$$

If $1<p<2$ and $\sqrt{1-p / 3} \leq t<\infty$ then for any $u$,

$$
\left\|\left(u^{2}+t^{2} v^{2}\right)^{1 / 2}\right\|_{p} \leq\left(1+t^{2} \tan ^{2} \frac{\pi}{2 p}\right)^{1 / 2}\|u\|_{p} .
$$

In each case the constant is sharp.

We remark that Theorems 5.2 and 5.3 may be interpreted as saying that

$$
\left\|I+i t H^{\mathbb{T}}\right\|_{L^{p}(\mathbb{T})}=\left(1+t^{2} \cot ^{2} \frac{\pi}{2 p^{*}}\right)^{1 / 2}
$$

if $p \geq 2$ or if $t \geq 1$, but it must then be remembered that $I+i t H^{\mathbb{T}}$ is considered as a hybrid operator from the real space $L^{p}(\mathbb{T})$ to the complex space $L^{p}(\mathbb{T})$.

Proof. The proof that the inequalities are sharp is essentially the same as in Theorem 5.1. 
The proof is very similar to that of Theorem 5.1. We assume $p \neq 2$. In this case we define

$$
\begin{aligned}
& c=\min _{-\pi \leq \theta<\pi} \frac{\Phi_{t}\left(e^{i \theta}\right)^{p}+\Phi_{t}\left(e^{i(\theta+\pi / p)}\right)^{p}}{\Phi_{0}\left(e^{i \theta}\right)^{p}+\Phi_{0}\left(e^{i(\theta+\pi / p)}\right)^{p}}, \\
& d=\max _{-\pi \leq \theta<\pi} \frac{\Phi_{t}\left(e^{i \theta}\right)^{p}+\Phi_{t}\left(e^{i(\theta+\pi / p)}\right)^{p}}{\Phi_{0}\left(e^{i \theta}\right)^{p}+\Phi_{0}\left(e^{i(\theta+\pi / p)}\right)^{p}} .
\end{aligned}
$$

Let

$$
F^{+}(z)=\Phi_{t}(z)^{p}-c|x|^{p}, \quad F^{-}(z)=d|x|^{p}-\Phi_{t}(z)^{p} .
$$

In place of (5.6) we use

$$
\Delta \Phi_{t}(z)^{p}=p\left(1+(p-1) t^{2}\right) \Phi_{t}(z)^{p-2}-p(p-2)\left(t^{2}-1\right)|x|^{2} \Phi_{t}(z)^{p-4} .
$$

For $x \neq 0$ let us introduce $\lambda(z)=\Phi_{t}(z) /|x|=\left(1+t^{2} y^{2} / x^{2}\right)^{1 / 2}$. Now for any choice of $a \in \mathbb{R}$,

$$
\begin{aligned}
& \Delta\left(\Phi_{t}(z)^{p}-a \Phi_{0}(z)^{p}\right) \\
& \quad=|x|^{p-2}\left(p\left(1+(p-1) t^{2}\right) \lambda^{p-2}-p(p-2)\left(t^{2}-1\right) \lambda^{p-4}-p(p-1) a\right) .
\end{aligned}
$$

Let

$\varphi(\lambda)=p\left(1+(p-1) t^{2}\right) \lambda^{p-2}-p(p-2)\left(t^{2}-1\right) \lambda^{p-4}-p(p-1) a, \quad 1 \leq \lambda<\infty$.

We show $\varphi(\lambda)$ vanishes at most once in $(1, \infty)$. In fact

$$
\varphi^{\prime}(\lambda)=p(p-2)\left(\left(1+(p-1) t^{2}\right) \lambda^{p-3}-(p-4)\left(t^{2}-1\right) \lambda^{p-5}\right)
$$

vanishes only when

$$
1-\lambda^{2}=\frac{p+3\left(t^{2}-1\right)}{1+(p-1) t^{2}}
$$

Thus if $p \geq 3$ or if $t \geq \sqrt{1-p / 3}$ then $\varphi^{\prime}$ can have no zeros in $(1, \infty)$ (for any choice of $a$ ) and so $\Delta F$ vanishes on at most one ray in the positive quadrant for $F=F^{+}$or $F=F^{-}$. This means we can apply Theorem 3.7 to produce a continuous $p$-homogeneous subharmonic minorant $G$.

Let us consider the case $2<p<\infty$ and $F=F^{+}$. In this case

$$
c=\left(1+t^{2} \tan ^{2} \frac{\pi}{2 p}\right)^{p / 2}
$$

by Corollary 3.8. Since $G(0)=0$ by homogeneity, we obtain (5.9) by the standard arguments.

Next we consider $1<p<2$ and $F=F^{-}$. Then

$$
d=\left(1+t^{2} \tan ^{2} \frac{\pi}{2 p}\right)^{p / 2}
$$

by Corollary 3.8 and $G$ is then of the form $G\left(r e^{i \theta}\right)=A r^{p} \cos p \theta$ if $-\frac{\pi}{2 p}<$ $\theta<\frac{\pi}{2 p}$ by the construction of $G$ in the proof of Theorem 3.5. In this case 
$F\left(e^{i \theta}\right)=G\left(e^{i \theta}\right)$ is negative for $\frac{\pi}{2 p}<\theta<\frac{\pi}{2 p}+\delta$ for some $\delta>0$ and so, since $G$ is subharmonic and $p$-homogeneous, $G\left(e^{i \theta}\right)>0$ for $-\frac{\pi}{2 p}<\theta<-\frac{\pi}{2 p}+\delta$, i.e. $A>0$. Hence $G$ is positive on the real axis. Then (5.10) follows.

We now discuss the extent to which Theorem 5.3 can hope to be improved. It is in fact clear that in the case $1<p<3$ the inequalities hold for some $t$ with $t<\sqrt{1-p / 3}$. More precisely one can show, with a little more work, that the inequalities hold if $t \geq \tau$ where $\tau=\tau(p)$ is the largest positive root of

$$
1+\frac{t^{2}}{p-1}=\left(1+t^{2} \tan ^{2} \frac{\pi}{2 p}\right)^{p / 2} .
$$

Here $\tau(p)$ is in general less than $\sqrt{1-p / 3}$. However these estimates are not best possible. The problem of determining the best possible value of $\tau$ is more delicate, and will be addressed below. We first show however that the inequalities in Theorem 5.3 do not hold for all $t>0$ when $1<p<3, p \neq 2$.

Proposition 5.4. (1) Suppose $1<p<2$; then there exists $t_{0}(p)>0$ such that the inequality

$$
\left\|\left(u^{2}+t^{2} v^{2}\right)^{1 / 2}\right\|_{p} \leq\left(1+t^{2} \tan ^{2} \frac{\pi}{2 p}\right)^{1 / 2}\|u\|_{p}
$$

fails if $0<t<t_{0}$.

(2) Suppose $2<p<3$; then there exists $t_{0}(p)>0$ such that the inequality

$$
\left\|\left(u^{2}+t^{2} v^{2}\right)^{1 / 2}\right\|_{p} \geq\left(1+t^{2} \tan ^{2} \frac{\pi}{2 p}\right)^{1 / 2}\|u\|_{p}
$$

fails if $0<t<t_{0}$.

Proof. We will need a simple trigonometric inequality. Note that the function

$$
h(\theta)=(\pi-\theta) \cos \theta-(\pi-2 \theta)
$$

is convex on the interval $\frac{\pi}{3} \leq \theta \leq \pi$ and that $h\left(\frac{\pi}{3}\right)=h\left(\frac{\pi}{2}\right)=0$ and $h(\pi)>0$. Hence

$$
\begin{array}{ll}
h(\theta)<0 & \text { if } \frac{\pi}{3}<\theta<\frac{\pi}{2}, \\
h(\theta)>0 & \text { if } \frac{\pi}{2}<\theta<\pi .
\end{array}
$$

If we put $\theta=\frac{\pi}{p}$ then these inequalities become

$$
\begin{array}{ll}
(p-1) \cos \frac{\pi}{p}<p-2 & \text { if } 2<p<3, \\
(p-1) \cos \frac{\pi}{p}>p-2 & \text { if } 1<p<2 .
\end{array}
$$


Hence

$$
\begin{array}{ll}
2(p-1) \cos ^{2} \frac{\pi}{2 p}<2 p-3 & \text { if } 2<p<3, \\
2(p-1) \cos ^{2} \frac{\pi}{2 p}>2 p-3 & \text { if } 1<p<2 .
\end{array}
$$

Finally this implies

$$
\begin{array}{ll}
(2 p-3) \tan ^{2} \frac{\pi}{2 p}<1 & \text { if } 1<p<2, \\
(2 p-3) \tan ^{2} \frac{\pi}{2 p}>1 & \text { if } 2<p<3 .
\end{array}
$$

We now turn to the two cases.

(1) We argue that if (5.12) holds for a particular choice of $t$ then

$$
F(z)=\left(1+t^{2} \tan ^{2} \frac{\pi}{2 p}\right)^{p / 2} \Phi_{0}(z)^{p}-\Phi_{t}(z)^{p}
$$

has the property that $\Delta F\left(e^{i \pi /(2 p)}\right) \geq 0$. This follows directly from Cole's theorem, Theorem 2.1, and the fact that $F(z)=F(\bar{z})$, since the existence of a subharmonic minorant implies that for all $z$,

$$
F\left(e^{-i \pi /(2 p)} z\right)+F\left(e^{i \pi /(2 p)} z\right) \geq 0
$$

However, we can also obtain (5.16) directly, avoiding the use of Cole's theorem by considering the functions $g_{\gamma}$ and $f_{\gamma}$ as in the proof of Theorem 5.1, but using $e^{i \theta} f_{\gamma}$ for all $0 \leq \theta<2 \pi$. Then if $F(z)=r^{p} f(\theta)$, we have $f\left(\frac{\pi}{2 p}\right)=0$, and (5.16) implies that $f\left(\theta-\frac{\pi}{2 p}\right)+f\left(\theta+\frac{\pi}{2 p}\right)$ has a minimum at $\theta=0$. Since $f$ is an even function it follows that $f^{\prime \prime}\left(\frac{\pi}{2 p}\right) \geq 0$, and using the fact that $f\left(\frac{\pi}{2 p}\right)=0$ we deduce that $\Delta F\left(e^{i \pi /(2 p)}\right) \geq 0$.

Now, if we assume $\Delta F\left(e^{i \pi /(2 p)}\right) \geq 0$ then by $(5.11)$,

$$
(p-1) \lambda^{4}-\left(1+(p-1) t^{2}\right) \lambda^{2}-(p-2)\left(1-t^{2}\right) \geq 0
$$

where $\lambda=\left(1+t^{2} \tan ^{2} \frac{\pi}{2 p}\right)^{1 / 2}$. This in turn implies

$$
(p-1)\left(\tan ^{4} \frac{\pi}{2 p}-\tan ^{2} \frac{\pi}{2 p}\right) t^{4}+\left((2 p-3) \tan ^{2} \frac{\pi}{2 p}-1\right) t^{2} \geq 0 .
$$

If this holds for arbitrarily small $t$ we have

This is false by (5.14).

$$
(2 p-3) \tan ^{2} \frac{\pi}{2 p} \geq 1 \text {. }
$$

(2) The calculations are similar but signs are reversed. We conclude in this case that the validity of (5.13) for arbitarily small $t$ implies that

Apply (5.15).

$$
(2 p-3) \tan ^{2} \frac{\pi}{2 p} \leq 1
$$


We now calculate the critical value $\tau=\tau(p)$ for (5.12).

TheOREm 5.5. Let $1<p<2$, and let $\tau(p)$ be the unique positive root of the equation

$$
\left(1+t^{2} \tan ^{2} \frac{\pi}{2 p}\right)^{1-p / 2}=1+t^{2} \tan ^{2} \frac{\pi}{2 p}\left(1-\frac{\cos ^{p-1} \frac{\pi}{2 p}}{\sin \frac{\pi}{2 p}}\right) .
$$

Then

$$
\left\|\left(u^{2}+t^{2} v^{2}\right)^{1 / 2}\right\|_{p} \leq\left(1+t^{2} \tan ^{2} \frac{\pi}{2 p}\right)^{1 / 2}\|u\|_{p}
$$

holds for $\tau(p) \leq t \leq 1$, but fails for $0<t<\tau(p)$.

Proof. Let

$$
\beta=1-\frac{\cos ^{p-1} \frac{\pi}{2 p}}{\sin \frac{\pi}{2 p}}, \quad \mu=\left(1+t^{2} \tan ^{2} \frac{\pi}{2 p}\right)^{1 / 2} .
$$

We need an elementary inequality,

$$
0<\beta<1-p / 2, \quad 1<p<2,
$$

which will follow from the analysis below. Then the function

$$
\psi(\mu)=\mu^{2-p}-1-\beta\left(\mu^{2}-1\right), \quad 1 \leq \mu<\infty,
$$

is concave on $(1, \infty)$ since $\psi^{\prime \prime}(\mu)=-(2-p)(p-1) \mu^{-p}-2 \beta<0$, and has a maximum at $\mu_{0}$ where

$$
\mu_{0}^{p}=\frac{2-p}{2 \beta}>1 .
$$

Since $\psi(1)=0$, there is a unique root $\mu_{1}>1$ of the equation $\psi(\mu)=0$, so that $\mu_{1}>\mu_{0}>1$. Consequently, (5.17) has only one positive root $t=\tau(p)$. To show that $\tau(p)<1$, notice that if $t=1$ then $\mu=1 / \cos \frac{\pi}{2 p}$, and the following elementary estimate holds:

$$
\psi(\mu)=\cos ^{p-2} \frac{\pi}{2 p}-1-\beta \tan ^{2} \frac{\pi}{2 p}=\frac{\cos ^{p-1} \frac{\pi}{2 p}\left(\cos \frac{\pi}{2 p}+\sin \frac{\pi}{2 p}\right)-1}{\cos ^{2} \frac{\pi}{2 p}}<0 .
$$

This yields $\tau(p)<1$. Again, a direct analytic proof of the preceding inequality is tedious, but we will show below that actually $\tau(p)<\sqrt{1-p / 3}<1$.

Let $d=\left(1+t^{2} \tan ^{2} \frac{\pi}{2 p}\right)^{p / 2}$. Notice that the function $\varphi(\lambda)=p\left(1+(p-1) t^{2}\right) \lambda^{p-2}-p(p-2)\left(t^{2}-1\right) \lambda^{p-4}-p(p-1) d, \quad 1 \leq \lambda<\infty$, vanishes at most twice in $(1, \infty)$. This follows from the fact already used above that

$$
\varphi^{\prime}(\lambda)=p(p-2)\left(\left(1+(p-1) t^{2}\right) \lambda^{p-3}-(p-4)\left(t^{2}-1\right) \lambda^{p-5}\right)
$$


vanishes only at $\lambda=\lambda_{0}$ where

$$
\lambda_{0}^{2}=\frac{(4-p)\left(1-t^{2}\right)}{1+(p-1) t^{2}}
$$

and $\lim _{\lambda \rightarrow \infty} \varphi(\lambda)=-p(p-1) d<0$. Note that $\lambda_{0} \in(1, \infty)$ if and only if $0<t<\sqrt{1-p / 3}$, and in this case $\varphi^{\prime}(\lambda)>0$ for $1<\lambda<\lambda_{0}$, and $\varphi^{\prime}(\lambda)<0$ for $\lambda_{0}<\lambda<\infty$.

If $F(z)=d \Phi_{0}(z)^{p}-\Phi_{t}(z)^{p}$, then (see the formula after (5.11))

$$
\Delta F(z)=-|x|^{p-2} \varphi(\lambda(z)), \quad \lambda(z)=\left(1+\frac{t^{2} y^{2}}{x^{2}}\right)^{1 / 2} .
$$

Hence by the discussion above $\Delta F(z)$ vanishes at most on two rays in the positive quadrant.

If $\Delta F(z)$ vanishes at most on one ray in the positive quadrant, then (5.18) follows by the same argument as in the proof of Theorem 5.3.

Thus it remains to consider the case where $\Delta F(z)$ vanishes exactly on two distinct rays $\arg z=\theta_{1}$ and $\arg z=\theta_{2}$ such that $0<\theta_{1}<\theta_{2}<\frac{\pi}{2}$. This may happen only if $0<t<\sqrt{1-p / 3}$ when we necessarily have $\varphi(1)=$ $p t^{2}-p(p-1)(d-1)<0$, and so $0<t<\min (\sqrt{(p-1)(d-1)}, \sqrt{1-p / 3})$.

Hence, for $z$ in the positive quadrant, we assume that $F(z)$ is superharmonic in the sector $\theta_{1}<\arg z<\theta_{2}<\frac{\pi}{2}$, and is subharmonic outside.

We next define a $p$-homogeneous function $G\left(r e^{i \theta}\right)=r^{p} g(\theta)$, where $g$ is even and $\pi$-periodic on $\mathbb{R}$, so that $G\left(r e^{i \theta}\right)=A r^{p} \cos p \theta$ for $|\theta| \leq \frac{\pi}{2 p}$, and $G\left(r e^{i \theta}\right)=F\left(r e^{i \theta}\right)$ for $\frac{\pi}{2 p}<|\theta| \leq \frac{\pi}{2}$. Here

$$
A=\cos ^{p-3} \frac{\pi}{2 p} \sin \frac{\pi}{2 p}\left(1+t^{2} \tan ^{2} \frac{\pi}{2 p}\right)^{p / 2-1} t^{2}
$$

is found from the equation $A=\frac{1}{p} f^{\prime}\left(-\frac{\pi}{2 p}\right)$, where

$$
f(\theta)=F\left(e^{i \theta}\right)=d|\cos \theta|^{p}-\left(\cos ^{2} \theta+t^{2} \sin ^{2} \theta\right)^{p / 2} .
$$

This guarantees that $F-G \in C^{1}(\mathbb{C})$.

Clearly, equation (5.17) can be rewritten in the form $A=d-1$.

We now prove that if $A \leq d-1$, then the function $G$ constructed above is a subharmonic minorant of $F$. Let $\Phi(z)=F(z)-G(z)$. Clearly, $\Phi(z)=\Phi(\bar{z})$. Notice that $\Phi$ vanishes on the rays $\arg z= \pm \frac{\pi}{2 p}$, and is nonnegative on the real axis.

As was explained above, it suffices to consider the case where $F$ is superharmonic in $0<\theta_{1}<\arg z<\theta_{2}<\frac{\pi}{2}$. We claim that actually

$$
0<\theta_{1}<\theta_{2}<\frac{\pi}{2 p} \text {. }
$$

Otherwise one of the following two cases holds. 
(1) If $\frac{\pi}{2 p} \leq \theta_{1}$, then $F$, and consequently $\Phi$, is subharmonic in the sector

$$
S=\left\{z \in \mathbb{C}:|\arg z|<\frac{\pi}{2 p}\right\} .
$$

Hence $\Phi\left(r e^{i \theta}\right)=r^{p} \phi(p \theta)$ where $\phi(\theta)$ is trigonometrically convex on $\left(-\frac{\pi}{2}, \frac{\pi}{2}\right)$, and $\phi\left( \pm \frac{\pi}{2}\right)=0$. Then by Proposition 3.4(2), $\phi$ is sinusoidal, and by Proposition $3.3, \Phi$ is harmonic in $S$, which is false.

(2) If $\theta_{1}<\frac{\pi}{2 p} \leq \theta_{2}$, then $\phi(\theta)$ is trigonometrically convex when $|\theta|<p \theta_{1}$, and trigonometrically concave when $p \theta_{1}<|\theta|<\frac{\pi}{2}$. The rest of the argument can be completed as in the proof of Proposition 3.4(3) using additionally the fact that $\phi$ is an even function.

Alternatively, we argue that $\phi\left( \pm \frac{\pi}{2}\right)=0, \phi^{\prime}\left( \pm \frac{\pi}{2}\right)=0$, and $\Delta \Phi\left(e^{ \pm i \pi / 2 p}\right)$ $=\phi^{\prime \prime}\left( \pm \frac{\pi}{2}\right)+p^{2} \phi\left( \pm \frac{\pi}{2}\right) \leq 0$ in the distributional sense. It follows that $\phi(\theta)$ has a maximum at $\pm \frac{\pi}{2}$, and so $\phi(\theta)<0$ in some neighborhood of $\theta= \pm \frac{\pi}{2}$. Thus $\Phi(z)$ is negative in some neighborhood of the rays $\arg z= \pm \frac{\pi}{2 p}$, but is positive on the positive real axis. Hence $\Phi(z)$ must vanish also on the rays $\arg z= \pm \widetilde{\theta}$ where $\widetilde{\theta} \in\left(0, \frac{\pi}{2 p}\right)$. If $\widetilde{\theta} \leq \theta_{1}$, then $\Phi$ must be subharmonic and nonnegative in a sector

$$
S^{\prime}=\{z \in \mathbb{C}:|\arg z|<\widetilde{\theta}\},
$$

where ang $S^{\prime}<\frac{\pi}{p}$, which contradicts the Phragmén-Lindelöf principle. sector

Similarly, if $\widetilde{\theta} \in\left(\theta_{1}, \frac{\pi}{2 p}\right)$, then $\Phi$ is superharmonic and nonpositive in a

$$
S^{\prime \prime}=\left\{z \in \mathbb{C}: \widetilde{\theta}<\arg z<\frac{\pi}{2 p}\right\}
$$

where ang $S^{\prime \prime}<\frac{\pi}{p}$, which again violates the Phragmén-Lindelöf principle. This proves (5.24), and consequently the fact that $G$ is subharmonic in $\mathbb{C}$.

We now prove $\Phi \geq 0$ for $z \in S$. Note that $\Phi(1)=F(1)-G(1)>0$ by $(5.23)$, and $\Phi(z)=\Phi(\bar{z})$. Hence $\Phi(z)$ cannot change sign in $S$. Otherwise there exists a subsector of $S$ where $\Phi$ is either subharmonic and nonnegative, or superharmonic and nonpositive, which violates the Phragmén-Lindelöf principle again. This completes the proof that $G$ is a subharmonic minorant of $F$, which is nonnegative on the real axis, in case $A \leq d-1$, and thus (5.18) holds in this case.

It is worth noting that, conversely, whenever $F$ has a $p$-homogeneous subharmonic minorant $G$ nonnegative on the real line it follows that $A \leq$ $d-1$. In fact, as was shown in the proof of Theorem 5.3, $G\left(r e^{i \theta}\right)=A r^{p} \cos p \theta$ for $|\theta| \leq \frac{\pi}{2 p}$, where $A$ is a positive constant. To verify that $A$ is given by (5.23), note that $g(\theta)=G\left(e^{i \theta}\right)$ is even, and $g(\theta / p)$ is trigonometrically convex. Hence by Lemma 3.1, $g(\theta) \geq A \cos p \theta$ if $\theta \in\left(-\frac{\pi}{2 p}-\delta,-\frac{\pi}{2 p}+\delta\right)$ for 
some $\delta>0$. Thus $F\left(r e^{i \theta}\right) \geq G\left(r e^{i \theta}\right) \geq A r^{p} \cos p \theta$ in the sector

$$
S_{\delta}=\left\{z:\left|\arg z+\frac{\pi}{2 p}\right|<\delta\right\} .
$$

Since $F\left(r e^{i \theta}\right)-A r^{p} \cos p \theta$ is nonnegative in $S_{\delta}$, and vanishes on $\arg z=$ $-\frac{\pi}{2 p}$, it follows that $F\left(e^{i \theta}\right)-A \cos p \theta$ has a minimum at $\theta=-\frac{\pi}{2 p}$. Hence $A=\frac{1}{p} f^{\prime}\left(-\frac{\pi}{2 p}\right)$, where $f(\theta)=F\left(e^{i \theta}\right)$, which coincides with (5.23). Thus $d-1=F(1) \geq G(1)=A$.

We remark that in the special case $t=1$ clearly $A \leq d-1$ since $F$ has a subharmonic minorant nonnegative on the real line as was shown in Theorem 5.3. This is equivalent to the estimate

$$
\left(1+\tan ^{2} \frac{\pi}{2 p}\right)^{1-p / 2} \leq 1+\tan ^{2} \frac{\pi}{2 p} \beta,
$$

where $\beta$ is defined in (5.19). In particular $\beta>0$ as was claimed above.

In fact $0<\beta<1-p / 2$ since in the case $\beta \geq 1-p / 2$ it follows that the concave function $\psi$ defined by $(5.21)$ is decreasing on $(1, \infty)$ and so $A \leq d-1$ for every $t>0$. This contradicts Proposition 5.4 where it is shown that $F$ does not have a subharmonic minorant nonnegative on the real axis if $0<t<t_{0}$. Thus the equation $\psi(\mu)=0$ has a unique root $\mu_{0}>1$. Consequently, the inequality $A \leq d-1$ is equivalent to $t \geq \tau(p)$, where the critical value $\tau(p)$ coincides with the unique positive root of (5.17). If $0<t<\tau(p)$ then $A>d-1$ and hence $F$ does not have a subharmonic minorant nonnegative on the real axis. Then by Cole's theorem (5.18) fails. It remains to notice that $0<\tau(p)<\sqrt{1-p / 3}<1$ by Proposition 5.4.

We conclude this section with a uniform estimate for the norm of the operator $(\cos t) I+(\sin t) H$ mentioned in the Introduction.

Theorem 5.6. Let $1<p<\infty$. Then

$$
\max _{0 \leq t \leq 2 \pi}\left\|(\cos t) I+(\sin t) H^{\mathbb{R}}\right\|_{L^{p}(\mathbb{R})}=\cot \frac{\pi}{2 p^{*}} .
$$

Proof. By Corollary 4.4,

$$
\begin{aligned}
\max _{0 \leq t \leq 2 \pi} \|(\cos t) I+(\sin t) & H^{\mathbb{R}} \|_{L^{p}(\mathbb{R})}^{p} \\
& =\max _{\substack{0 \leq t \leq 2 \pi \\
0 \leq \theta \leq 2 \pi}} \frac{|\cos (\theta+t)|^{p}+\left|\cos \left(\theta+t+\frac{\pi}{p}\right)\right|^{p}}{|\cos \theta|^{p}+\left|\cos \left(\theta+\frac{\pi}{p}\right)\right|^{p}} \\
& =\frac{\max _{0 \leq t \leq 2 \pi}\left(|\cos t|^{p}+\left|\cos \left(t+\frac{\pi}{p}\right)\right|^{p}\right)}{\min _{0 \leq \theta \leq 2 \pi}\left(|\cos \theta|^{p}+\left|\cos \left(\theta+\frac{\pi}{p}\right)\right|^{p}\right)}=\lambda_{p} \mu_{p},
\end{aligned}
$$


where

$$
\begin{aligned}
\lambda_{p} & =\max _{0 \leq t \leq 2 \pi}\left(|\cos t|^{p}+\left|\cos \left(t+\frac{\pi}{p}\right)\right|^{p}\right), \\
\mu_{p}^{-1} & =\min _{0 \leq \theta \leq 2 \pi}\left(|\cos \theta|^{p}+\left|\cos \left(\theta+\frac{\pi}{p}\right)\right|^{p}\right) .
\end{aligned}
$$

It is not difficult to show directly that

$$
\lambda_{p}=2 \cos ^{p} \frac{\pi}{2 p^{*}}, \quad \mu_{p}^{-1}=2 \sin ^{p} \frac{\pi}{2 p^{*}} .
$$

However it is easier to verify this using the proof of Theorem 5.3 where it was shown that, in the case $t=1$,

$$
c=\max _{0 \leq \theta \leq 2 \pi} \frac{2}{|\cos \theta|^{p}+\left|\cos \left(\theta+\frac{\pi}{p}\right)\right|^{p}}=\frac{1}{\sin ^{p} \frac{\pi}{2 p^{*}}}
$$

is the best constant in the inequality

$$
\left\|\left(u^{2}+v^{2}\right)^{1 / 2}\right\|_{p}^{p} \leq c\|u\|_{p}^{p}
$$

and

$$
d=\frac{1}{2} \max _{0 \leq t \leq 2 \pi}\left(|\cos t|^{p}+\left|\cos \left(t+\frac{\pi}{p}\right)\right|^{p}\right)=\cos ^{p} \frac{\pi}{2 p^{*}}
$$

is the best constant in the inequality

$$
\|u\|_{p}^{p} \leq d\left\|\left(u^{2}+v^{2}\right)^{1 / 2}\right\|_{p}^{p}
$$

for $u$ with mean zero. Since $\lambda_{p}=2 d$ and $\mu_{p}=c / 2$, it follows that $\lambda_{p} \mu_{p}=$ $\cot ^{p} \frac{\pi}{2 p^{*}}$.

6. The norm of $I-\Pi$. Let us define the re-expansion operator, $\Pi=$ $\mathcal{F}_{\mathrm{s}} \mathcal{F}_{\mathrm{c}}$, where $\mathcal{F}_{\mathrm{s}}$ and $\mathcal{F}_{\mathrm{c}}$ are the sine and cosine Fourier transforms on the positive real axis $\mathbb{R}_{+}=(0, \infty)$ :

$$
\mathcal{F}_{\mathrm{c}} f(x)=\sqrt{\frac{2}{\pi}} \int_{\mathbb{R}_{+}} f(t) \cos t x d t, \quad \mathcal{F}_{\mathrm{s}} f(x)=\sqrt{\frac{2}{\pi}} \int_{\mathbb{R}_{+}} f(t) \sin t x d t .
$$

We will also need the complex Fourier transform in the upper half-plane,

$$
\mathcal{F} f(z)=\sqrt{\frac{2}{\pi}} \int_{0}^{\infty} f(t) e^{i z t} d t, \quad z=x+i y, y \geq 0,
$$

and the Laplace transform,

$$
\mathcal{L} f(y)=\sqrt{\frac{2}{\pi}} \int_{0}^{\infty} f(t) e^{-y t} d t, \quad y \geq 0
$$


In the field of scattering theory it is of interest to examine the operator $I-\Pi$. Using Theorem 4.1, we will compute the exact value of $\|I-\Pi\|_{L^{p}\left(\mathbb{R}_{+}\right)}$ for $1<p<\infty$.

We denote by $L^{p}\left(\mathbb{R}_{+}, x^{\alpha}\right), \alpha>-1$, the space of measurable functions $f$ on $\mathbb{R}_{+}$such that

$$
\|f\|_{L^{p}\left(\mathbb{R}_{+}, x^{\alpha}\right)}^{p}=\int_{\mathbb{R}_{+}}|f(x)|^{p} x^{\alpha} d x<\infty .
$$

As was mentioned in the Introduction,

$$
\Pi f(x)=\frac{1}{\pi} \mathrm{p} \cdot \mathrm{v} \cdot \int_{\mathbb{R}_{+}} \frac{2 x f(t)}{x^{2}-t^{2}} d t,
$$

from which it follows that $\Pi=H^{\mathbb{R}_{+}}+H_{1}$, where

$$
H^{\mathbb{R}_{+}} f(x)=\frac{1}{\pi} \text { p.v. } \int_{\mathbb{R}_{+}} \frac{f(t)}{x-t} d t, \quad H_{1} f(x)=\frac{1}{\pi} \int_{\mathbb{R}_{+}} \frac{f(t)}{x+t} d t .
$$

Note that $H_{1}=\frac{1}{2} \mathcal{L}^{2}$. The formal adjoint operator $\Pi^{*}=\mathcal{F}_{\mathrm{c}} \mathcal{F}_{\mathrm{s}}$ is given by

$$
\Pi^{*} f(x)=-\frac{1}{\pi} \text { p.v. } \int_{\mathbb{R}_{+}} \frac{2 t f(t)}{x^{2}-t^{2}} d t .
$$

We first consider the case $p=2$.

Lemma 6.1. Let $f$ be a real-valued function in $L^{2}\left(\mathbb{R}_{+}\right)$. Then

$$
\left\|\mathcal{F}_{\mathrm{c}} f \pm \mathcal{F}_{\mathrm{s}} f\right\|_{L^{2}\left(\mathbb{R}_{+}\right)}^{2}=2\|f\|_{L^{2}\left(\mathbb{R}_{+}\right)}^{2} \pm\|\mathcal{L} f\|_{L^{2}\left(\mathbb{R}_{+}\right)}^{2},
$$

and

$$
\|I-\Pi\|_{L^{2}\left(\mathbb{R}_{+}\right)}=\sqrt{2}, \quad\|I+\Pi\|_{L^{2}\left(\mathbb{R}_{+}\right)}=2 .
$$

Proof. Clearly,

$$
\begin{aligned}
\left\|\mathcal{F}_{\mathrm{c}} f \pm \mathcal{F}_{\mathrm{s}} f\right\|_{L^{2}\left(\mathbb{R}_{+}\right)}^{2} & =\left\|\mathcal{F}_{\mathrm{c}} f\right\|_{L^{2}\left(\mathbb{R}_{+}\right)}^{2}+\left\|\mathcal{F}_{\mathrm{s}} f\right\|_{L^{2}\left(\mathbb{R}_{+}\right)}^{2} \pm 2\left\langle\mathcal{F}_{\mathrm{c}} f, \mathcal{F}_{\mathrm{s}} f\right\rangle \\
& =2\|f\|_{L^{2}\left(\mathbb{R}_{+}\right)}^{2} \pm 2\left\langle\mathcal{F}_{\mathrm{c}} f, \mathcal{F}_{\mathrm{s}} f\right\rangle .
\end{aligned}
$$

Next, we notice that $\left\langle H^{\mathbb{R}_{+}} f, f\right\rangle=0$, and $\left\langle H_{1} f, f\right\rangle=\frac{1}{2}\|\mathcal{L} f\|_{L^{2}\left(\mathbb{R}_{+}\right)}^{2}$, and hence, by (6.1) and (6.2),

$$
\left\langle\mathcal{F}_{\mathrm{c}} f, \mathcal{F}_{\mathrm{s}} f\right\rangle=\left\langle H^{\mathbb{R}_{+}} f, f\right\rangle+\left\langle H_{1} f, f\right\rangle=\frac{1}{2}\|\mathcal{L} f\|_{L^{2}\left(\mathbb{R}_{+}\right)}^{2} \geq 0 .
$$

From this we have

$$
\begin{aligned}
& \|(I-\Pi) f\|_{L^{2}\left(\mathbb{R}_{+}\right)}^{2}=\left\|\mathcal{F}_{\mathrm{s}} f-\mathcal{F}_{\mathrm{c}} f\right\|_{L^{2}\left(\mathbb{R}_{+}\right)}^{2} \leq 2\|f\|_{L^{2}\left(\mathbb{R}_{+}\right)}^{2}, \\
& \|(I+\Pi) f\|_{L^{2}\left(\mathbb{R}_{+}\right)}^{2}=\left\|\mathcal{F}_{\mathrm{s}} f+\mathcal{F}_{\mathrm{c}} f\right\|_{L^{2}\left(\mathbb{R}_{+}\right)}^{2} \leq 4\|f\|_{L^{2}\left(\mathbb{R}_{+}\right)}^{2} .
\end{aligned}
$$

This proves $\|I-\Pi\|_{L^{2}\left(\mathbb{R}_{+}\right)} \leq \sqrt{2}$, and $\|I+\Pi\|_{L^{2}\left(\mathbb{R}_{+}\right)} \leq 2$. The lower estimates follow, for instance, from the fact that the spectrum of $\Pi$ in $L^{2}\left(\mathbb{R}_{+}\right)$is the semicircle $\{z:|z|=1, \operatorname{Re} z \geq 0\}$ (see [Bir]). Hence, the corresponding 
spectral radii are $\varrho(I-\Pi)=\sqrt{2}$ and $\varrho(I+\Pi)=2$, which completes the proof of (6.5).

We now discuss a connection with some inequalities in $L^{p}\left(\mathbb{R}_{+}\right)$with weights.

Lemma 6.2. Let $1<p<\infty$, and $p^{\prime}=p /(p-1)$. Then

$$
\begin{aligned}
\|I \pm \Pi\|_{L^{p}\left(\mathbb{R}_{+}\right)} & \left.=\left\|I \pm H^{\mathbb{R}_{+}}\right\|_{L^{p}\left(\mathbb{R}_{+}, x(p-1) / 2\right.}\right) \\
& =\left\|I \mp H^{\mathbb{R}_{+}}\right\|_{L^{p}\left(\mathbb{R}_{+}, x^{(p-3) / 2}\right)} \\
& =\left\|I \mp H^{\mathbb{R}_{+}}\right\|_{L^{p^{\prime}}\left(\mathbb{R}_{+}, x^{-1 / 2}\right)} \\
\left\|I \pm \Pi^{*}\right\|_{L^{p}\left(\mathbb{R}_{+}\right)} & =\left\|I \mp H^{\mathbb{R}_{+}}\right\|_{L^{p}\left(\mathbb{R}_{+}, x^{-1 / 2}\right)}=\left\|I \pm H^{\mathbb{R}_{+}}\right\|_{L^{p}\left(\mathbb{R}_{+}, x^{p-3 / 2}\right)} \\
& =\left\|I \pm H^{\mathbb{R}_{+}}\right\|_{L^{p^{\prime}}\left(\mathbb{R}_{+}, x^{\left(p^{\prime}-1\right) / 2}\right)}
\end{aligned}
$$

Proof. Define the isometry $J: L^{p}\left(\mathbb{R}_{+}\right) \rightarrow L^{p}\left(\mathbb{R}_{+}, x^{(p-1) / 2}\right)$ by

$$
J g(x)=2^{-1 / p} x^{-1 / 2} g(\sqrt{x}) .
$$

Then $J^{-1}: L^{p}\left(\mathbb{R}_{+}, x^{(p-1) / 2}\right) \rightarrow L^{p}\left(\mathbb{R}_{+}\right)$, where

$$
J^{-1} g(t)=2^{1 / p} \operatorname{tg}\left(t^{2}\right) \text {. }
$$

A substitution $u=x^{2}, v=t^{2}$ in (6.1) shows that $\Pi=J^{-1} H^{\mathbb{R}_{+}} J$, which yields

$$
\|I \pm \Pi\|_{L^{p}\left(\mathbb{R}_{+}\right)}=\left\|I \pm H^{\mathbb{R}_{+}}\right\|_{L^{p}\left(\mathbb{R}_{+}, x^{(p-1) / 2}\right)} .
$$

From this by duality

$$
\|I \pm \Pi\|_{L^{p}\left(\mathbb{R}_{+}\right)}=\left\|I \mp H^{\mathbb{R}_{+}}\right\|_{L^{p^{\prime}}\left(\mathbb{R}_{+}, x^{-1 / 2}\right)} .
$$

Similarly, one verifies

$$
\left\|I \pm \Pi^{*}\right\|_{L^{p}\left(\mathbb{R}_{+}\right)}=\left\|I \mp H^{\mathbb{R}_{+}}\right\|_{L^{p}\left(\mathbb{R}_{+}, x^{-1 / 2}\right)}=\left\|I \pm H^{\mathbb{R}_{+}}\right\|_{L^{p^{\prime}}\left(\mathbb{R}_{+}, x^{\left(p^{\prime}-1\right) / 2}\right)} .
$$

Also, using the change of variables $u=1 / x, v=1 / t$ in the first equation of $(6.2)$, it is easy to see that

$$
\begin{aligned}
\left\|I \pm H^{\mathbb{R}_{+}}\right\|_{L^{p}\left(\mathbb{R}_{+}, x^{(p-1) / 2}\right)} & =\left\|I \mp H^{\mathbb{R}_{+}}\right\|_{L^{p}\left(\mathbb{R}_{+}, x^{(p-3) / 2}\right)} \\
\left\|I \pm H^{\mathbb{R}_{+}}\right\|_{L^{p}\left(\mathbb{R}_{+}, x^{-1 / 2}\right)} & =\left\|I \mp H^{\mathbb{R}_{+}}\right\|_{L^{p}\left(\mathbb{R}_{+}, x^{p-3 / 2}\right)} \cdot
\end{aligned}
$$

We next give lower estimates of $\|I \pm \Pi\|_{L^{p}\left(\mathbb{R}_{+}\right)}$and $\left\|I \pm \Pi^{*}\right\|_{L^{p}\left(\mathbb{R}_{+}\right)}$in terms of the unweighted norms of $I \pm H^{\mathbb{R}_{+}}$.

Lemma 6.3. Let $1<p<\infty$. Then

$$
\begin{aligned}
\|I \pm \Pi\|_{L^{p}\left(\mathbb{R}_{+}\right)} & \geq\left\|I \pm H^{\mathbb{R}_{+}}\right\|_{L^{p}\left(\mathbb{R}_{+}\right)}=A_{p} \\
\left\|I \pm \Pi^{*}\right\|_{L^{p}\left(\mathbb{R}_{+}\right)} & \geq\left\|I \pm H^{\mathbb{R}_{+}}\right\|_{L^{p}\left(\mathbb{R}_{+}\right)}=A_{p} .
\end{aligned}
$$

Here $A_{p}=A_{p^{\prime}}=\left\|I \pm H^{\mathbb{R}}\right\|_{L^{p}(\mathbb{R})}$ is defined by (1.2). 
Proof. We first note that

$$
\left\|I \pm H^{\mathbb{R}_{+}}\right\|_{L^{p}\left(\mathbb{R}_{+}\right)}=\left\|I \pm H^{\mathbb{R}}\right\|_{L^{p}(\mathbb{R})} .
$$

This is a consequence of a more general fact that, if $a \in L^{\infty}(\mathbb{R})$, then the Fourier multiplier operator $M_{a} f=(a \widehat{f})^{\vee}$, defined initially on $L^{p}(\mathbb{R}) \cap$ $L^{2}(\mathbb{R})$, is bounded on $L^{p}(\mathbb{R})$ if and only if the Wiener-Hopf operator $W_{a}=$ $\chi_{\mathbb{R}_{+}} M_{a} \chi_{\mathbb{R}_{+}}$is bounded on $L^{p}\left(\mathbb{R}_{+}\right)$, and the corresponding operator norms coincide:

$$
\left\|M_{a}\right\|_{L^{p}(\mathbb{R})}=\left\|W_{a}\right\|_{L^{p}\left(\mathbb{R}_{+}\right)} .
$$

(See, e.g., [GK2], [St].) Another well known result on Fourier multipliers,

$$
\left\|M_{a}\right\|_{L^{p}(\mathbb{R})}=\left\|M_{\bar{a}}\right\|_{L^{p}(\mathbb{R})},
$$

in the special case $a(\xi)=1 \pm i \operatorname{sign} \xi$ yields

$$
\left\|I+H^{\mathbb{R}}\right\|_{L^{p}(\mathbb{R})}=\left\|I-H^{\mathbb{R}}\right\|_{L^{p}(\mathbb{R})} .
$$

By Corollary 4.4 with $a=1$ and $b= \pm 1$, this gives $\left\|I \pm H^{\mathbb{R}}\right\|_{L^{p}(\mathbb{R})}=A_{p}$. Since by duality

$$
\left\|I \pm H^{\mathbb{R}}\right\|_{L^{p}(\mathbb{R})}=\left\|I \mp H^{\mathbb{R}}\right\|_{L^{p^{\prime}}(\mathbb{R})},
$$

it follows that $A_{p}=A_{p^{\prime}}$, which is also not difficult to prove directly.

It remains to prove the lower estimates

$$
\begin{aligned}
\|I \pm \Pi\|_{L^{p}\left(\mathbb{R}_{+}\right)} & \geq\left\|I \pm H^{\mathbb{R}_{+}}\right\|_{L^{p}\left(\mathbb{R}_{+}\right)}, \\
\left\|I \pm \Pi^{*}\right\|_{L^{p}\left(\mathbb{R}_{+}\right)} & \geq\left\|I \pm H^{\mathbb{R}_{+}}\right\|_{L^{p}\left(\mathbb{R}_{+}\right)} .
\end{aligned}
$$

By (6.2), $I \pm \Pi=I \pm\left(H^{\mathbb{R}_{+}}+H_{1}\right)$, where $H_{1}$ is a Hankel operator with the kernel $\frac{1}{\pi(x+t)}$.

Denote by $U_{s}, s \geq 0$, the shift operator: $U_{s} f(t)=0$ if $0 \leq t \leq s$, and $U_{s} f(t)=f(t-s)$ if $t \geq s$ on $L^{p}\left(\mathbb{R}_{+}\right)$, and by $U_{-s}$ the backward shift $U_{-s} f(t)=f(t+s)$. Then obviously, $U_{-s} W_{a} U_{s}=W_{a}$ for every Wiener-Hopf operator $W_{a}$ with symbol $a$ on $\mathbb{R}$, while $U_{-s} H_{b} U_{s}=U_{-2 s} H_{b}$ for every Hankel operator $H_{b}$ with symbol $b$ (see $\left.[\mathrm{N}]\right)$. Hence

$$
\left\|W_{a}+H_{b}\right\|_{L^{p}\left(\mathbb{R}_{+}\right)} \geq\left\|U_{-s}\left(W_{a}+H_{b}\right) U_{s}\right\|_{L^{p}\left(\mathbb{R}_{+}\right)}=\left\|W_{a}+U_{-2 s} H_{a}\right\|_{L^{p}\left(\mathbb{R}_{+}\right)} .
$$

Letting $s \rightarrow \infty$ and using the fact that $U_{-s} \rightarrow 0$ in strong operator topology in $L^{p}\left(\mathbb{R}_{+}\right)$, we obtain

$$
\left\|W_{a}+H_{b}\right\|_{L^{p}\left(\mathbb{R}_{+}\right)} \geq\left\|W_{a}\right\|_{L^{p}\left(\mathbb{R}_{+}\right)}
$$

for any Wiener-Hopf operator $W_{a}$ and Hankel operator $H_{b}$ bounded on $L^{p}\left(\mathbb{R}_{+}\right)$. Applying the preceding estimate with $W_{a}=I \pm H^{\mathbb{R}_{+}}$and $H_{b}=$ $\pm H_{1}$, we obtain (6.13).

In the next lemma, we show how to find the norms $\|\Pi\|_{L^{p}\left(\mathbb{R}_{+}\right)}$for $1<$ $p<\infty$, and $\|I+\Pi\|_{L^{p}\left(\mathbb{R}_{+}\right)}$for $p \leq 2$, which is essentially a consequence of 
the known results (cf. [GK2]). We also give a new formula for $\|I+\Pi\|_{L^{p}\left(\mathbb{R}_{+}\right)}$ in the case $p \geq 3$.

Lemma 6.4. Let $p^{*}=\max \left(p, p^{\prime}\right)$. Then the following statements hold:

$$
\begin{aligned}
\|\Pi\|_{L^{p}\left(\mathbb{R}_{+}\right)} & =\left\|\Pi^{*}\right\|_{L^{p}\left(\mathbb{R}_{+}\right)}=\cot \frac{\pi}{2 p^{*}}, & & 1<p<\infty . \\
\|I+\Pi\|_{L^{p}\left(\mathbb{R}_{+}\right)} & =1+\tan \frac{\pi}{2 p}, & & 1<p \leq 2, \\
\left\|I+\Pi^{*}\right\|_{L^{p}\left(\mathbb{R}_{+}\right)} & =1+\cot \frac{\pi}{2 p} & & 2 \leq p<\infty, \\
\|I+\Pi\|_{L^{p}\left(\mathbb{R}_{+}\right)} & =A_{p}, & & 3 \leq p<\infty, \\
\left\|I+\Pi^{*}\right\|_{L^{p}\left(\mathbb{R}_{+}\right)} & =A_{p}, & & 1<p \leq 3 / 2,
\end{aligned}
$$

where $A_{p}$ is defined by (1.2).

Proof. Let $a(\xi)=-i \operatorname{sign} \xi$. Then $W_{a}=H^{\mathbb{R}_{+}}$and $H_{a}=H_{1}$. From (6.14) it follows that

$$
\|\Pi\|_{L^{p}\left(\mathbb{R}_{+}\right)}=\left\|H^{\mathbb{R}_{+}}+H_{1}\right\|_{L^{p}\left(\mathbb{R}_{+}\right)} \geq\left\|H^{\mathbb{R}_{+}}\right\|_{L^{p}\left(\mathbb{R}_{+}\right)} .
$$

Using (6.11) and the well known expression for the norm of the Hilbert transform, we get

$$
\left\|H^{\mathbb{R}_{+}}\right\|_{L^{p}\left(\mathbb{R}_{+}\right)}=\left\|H^{\mathbb{R}}\right\|_{L^{p}(\mathbb{R})}=\cot \frac{\pi}{2 p^{*}} .
$$

To prove the upper estimate, let $\widetilde{f}(x)=f(x)$ if $x \geq 0$, and $\widetilde{f}(x)=f(-x)$ if $x<0$. Then

$$
\|\Pi f\|_{L^{p}\left(\mathbb{R}_{+}\right)}^{p}=\frac{1}{2}\left\|H^{\mathbb{R}} \widetilde{f}\right\|_{L^{p}(\mathbb{R})}^{p} \leq \cot ^{p} \frac{\pi}{2 p^{*}}\|f\|_{L^{p}\left(\mathbb{R}_{+}\right)}^{p} .
$$

Hence, $\|\Pi\|_{L^{p}\left(\mathbb{R}_{+}\right)}=\cot \frac{\pi}{2 p^{*}}$, and by duality the same formula gives the norm of $\Pi^{*}$. This proves $(6.15)$.

Now we prove (6.16) and (6.17). Let $1<p \leq 2$. The upper estimate

$$
\|I+\Pi\|_{L^{p}\left(\mathbb{R}_{+}\right)} \leq 1+\|\Pi\|_{L^{p}\left(\mathbb{R}_{+}\right)}=1+\tan \frac{\pi}{2 p}
$$

follows from (6.15). To prove the lower estimate, we use some facts from the spectral theory of singular integral operators. Recall that, as was shown in the proof of Lemma $6.2, I+\Pi=J^{-1}\left(I-H^{\mathbb{R}_{+}}\right) J$, where $J$ is an isometry from $L^{p}\left(\mathbb{R}_{+}\right)$onto $L^{p}\left(\mathbb{R}_{+}, x^{(p-1) / 2}\right)$. Hence the spectrum of $I+\Pi$ in $L^{p}\left(\mathbb{R}_{+}\right)$ coincides with that of $I+H^{\mathbb{R}_{+}}$in $L^{p}\left(\mathbb{R}_{+}, x^{(p-1) / 2}\right)$.

For $1<p \leq 2$, the latter is known to be the larger arc of the circle

$$
\left|z-1+\cot \frac{\pi}{p}\right|=\csc \frac{\pi}{p}
$$

in the complex plane with the endpoints at $1 \pm i$. (The easiest way to see this is to map $\mathbb{R}_{+}$to $\mathbb{R}$ using an exponential substitution, and notice that 
$I+H^{\mathbb{R}_{+}}$on $L^{p}\left(\mathbb{R}_{+}, x^{(p-1) / 2}\right)$ is transformed to a Fourier multiplier on $L^{p}(\mathbb{R})$ which takes values exactly on the arc described above. See details in [GK2, Sec. 9.7].)

Hence, the spectral radius of $I+\Pi$ in $L^{p}\left(\mathbb{R}_{+}\right)$for $1<p \leq 2$ is given by

$$
\varrho(I+\Pi)=1-\cot \frac{\pi}{p}+\csc \frac{\pi}{p}=1+\tan \frac{\pi}{2 p} .
$$

This yields the lower estimate

$$
\|I+\Pi\|_{L^{p}\left(\mathbb{R}_{+}\right)} \geq \varrho(I+\Pi)=1+\tan \frac{\pi}{2 p} .
$$

This proves (6.16). The corresponding result for $\left\|I+\Pi^{*}\right\|_{L^{p}\left(\mathbb{R}_{+}\right)}$for $2 \leq p<\infty$ follows by duality, which proves (6.17).

To prove (6.18) and (6.19), notice that by Lemma 6.3,

$$
\|I+\Pi\|_{L^{p}\left(\mathbb{R}_{+}\right)} \geq\left\|I+H^{\mathbb{R}_{+}}\right\|_{L^{p}\left(\mathbb{R}_{+}\right)}=A_{p} .
$$

On the other hand, by Lemma 6.2,

$$
\|I+\Pi\|_{L^{p}\left(\mathbb{R}_{+}\right)}=\left\|I+H^{\mathbb{R}_{+}}\right\|_{L^{p}\left(\mathbb{R}_{+}, x^{(p-1) / 2}\right)} .
$$

Using the change of variables $u=1 / x, v=1 / t$ as in the proof of Lemma 6.2, we obtain

$$
A_{p}=\left\|I \pm H^{\mathbb{R}_{+}}\right\|_{L^{p}\left(\mathbb{R}_{+}\right)}=\left\|I \mp H^{\mathbb{R}_{+}}\right\|_{L^{p}\left(\mathbb{R}_{+}, x^{p-2}\right)} .
$$

Now let $p \geq 3$. Then $0<(p-1) / 2 \leq p-2$, and by interpolation in $L^{p}$ with a change of weights,

$$
\left\|I+H^{\mathbb{R}_{+}}\right\|_{L^{p}\left(\mathbb{R}_{+}, x^{(p-1) / 2}\right)} \leq\left\|I+H^{\mathbb{R}_{+}}\right\|_{L^{p}\left(\mathbb{R}_{+}\right)}^{1-t}\left\|I+H^{\mathbb{R}_{+}}\right\|_{L^{p}\left(\mathbb{R}_{+}, x^{p-2}\right)}^{t}=A_{p},
$$

where $t=\frac{p-1}{2(p-2)} \in(0,1)$, which proves the upper estimate.

Hence $\|I+\Pi\|_{L^{p}\left(\mathbb{R}_{+}\right)}=A_{p}$ for $p \geq 3$, i.e. (6.18) holds, while duality yields $\left\|I+\Pi^{*}\right\|_{L^{p}\left(\mathbb{R}_{+}\right)}=A_{p}$ for $1<p \leq 3 / 2$. This proves (6.19).

We are now in a position to prove the main theorem.

TheOREM 6.5. Let $1<p<\infty$, and let $\Pi$ be the re-expansion operator defined above. Then

$$
\|I-\Pi\|_{L^{p}\left(\mathbb{R}_{+}\right)}=\left\|I-\Pi^{*}\right\|_{L^{p}\left(\mathbb{R}_{+}\right)}=A_{p},
$$

where

$$
A_{p}=\sqrt{2} \max _{0 \leq \theta \leq 2 \pi}\left[\frac{\left|\cos \left(\theta-\frac{\pi}{4}\right)\right|^{p}+\left|\cos \left(\theta-\frac{\pi}{4}+\frac{\pi}{p}\right)\right|^{p}}{|\cos \theta|^{p}+\left|\cos \left(\theta+\frac{\pi}{p}\right)\right|^{p}}\right]^{1 / p} .
$$

Proof. By Lemma 6.3,

$$
\|I-\Pi\|_{L^{p}\left(\mathbb{R}_{+}\right)} \geq A_{p}, \quad\left\|I-\Pi^{*}\right\|_{L^{p}\left(\mathbb{R}_{+}\right)} \geq A_{p} .
$$

It remains to prove the upper estimate

$$
\|I-\Pi\|_{L^{p}\left(\mathbb{R}_{+}\right)} \leq A_{p},
$$


since a similar inequality for $I-\Pi^{*}$ follows by duality, and the equation $A_{p}=A_{p^{\prime}}$ (see Lemma 6.3).

Recall that $\Pi=\mathcal{F}_{\mathrm{s}} \mathcal{F}_{\mathrm{c}}, \mathcal{F}_{\mathrm{s}}^{2}=I$, and $\mathcal{F}_{\mathrm{c}}^{2}=I$. Let $B_{p}=A_{p}^{p}$. Clearly, (6.23) is equivalent to the estimate

$$
\left\|\mathcal{F}_{\mathrm{c}} f-\mathcal{F}_{\mathrm{s}} f\right\|_{L^{p}\left(\mathbb{R}_{+}\right)}^{p} \leq B_{p}\left\|\mathcal{F}_{\mathrm{c}} f\right\|_{L^{p}\left(\mathbb{R}_{+}\right)}^{p} .
$$

Here we assume that $f=\mathcal{F}_{\mathrm{c}} g$, where $g \in C_{0}^{\infty}\left(\mathbb{R}_{+}\right)$; then the complex Fourier transform $\mathcal{F} f(z)$ obviously satisfies the estimates

$$
|\mathcal{F} f(z)| \leq \frac{c}{1+|z|}, \quad\left|(\mathcal{F} f)^{\prime}(z)\right| \leq \frac{c}{1+|z|^{2}},
$$

which suffices to justify Green's formula for the positive quadrant used below.

We first consider a simpler case $p \geq 2$. We will need the inequality

$$
|x-y|^{p} \leq B_{p}|x|^{p}-G(x, y),
$$

where $G(x, y)$ is the subharmonic minorant for the function

$$
\Phi(x, y)=B_{p}|x|^{p}-|x-y|^{p}
$$

constructed in Lemma 4.2.

Let

$$
\begin{aligned}
& u(x, y)=\operatorname{Re} \mathcal{F} f(z)=\sqrt{\frac{2}{\pi}} \int_{0}^{\infty} f(t) \cos t x e^{-y t} d t \\
& v(x, y)=\operatorname{Im} \mathcal{F} f(z)=\sqrt{\frac{2}{\pi}} \int_{0}^{\infty} f(t) \sin t x e^{-y t} d t .
\end{aligned}
$$

Then, clearly,

$$
\begin{aligned}
& \frac{\partial u}{\partial x}(x, y)=-\sqrt{\frac{2}{\pi}} \int_{0}^{\infty} t f(t) \sin t x e^{-y t} d t \\
& \frac{\partial v}{\partial x}(x, y)=\sqrt{\frac{2}{\pi}} \int_{0}^{\infty} t f(t) \cos t x e^{-y t} d t .
\end{aligned}
$$

In particular, we will need the following relations:

$$
\begin{aligned}
u(x, 0) & =\mathcal{F}_{\mathrm{c}} f(x), & v(x, 0) & =\mathcal{F}_{\mathrm{s}} f(x), \\
u(0, y) & =\mathcal{L} f(y), & v(0, y) & =0,
\end{aligned}
$$

and

$$
\begin{aligned}
& \frac{\partial u}{\partial x}(0, y)=0 \\
& \frac{\partial v}{\partial x}(0, y)=-\frac{d(\mathcal{L} f)}{d y}(y)=\sqrt{\frac{2}{\pi}} \int_{0}^{\infty} t f(t) e^{-y t} d t .
\end{aligned}
$$


Putting $u(x, 0)$ and $v(x, 0)$ in place of $x$ and $y$ in $(6.26)$, and integrating over $\mathbb{R}_{+}$, we obtain

$$
\int_{\mathbb{R}_{+}}\left|\mathcal{F}_{\mathrm{c}} f(x)-\mathcal{F}_{\mathrm{s}} f(x)\right|^{p} d x \leq B_{p} \int_{\mathbb{R}_{+}}\left|\mathcal{F}_{\mathrm{c}} f(x)\right|^{p} d x-\int_{\mathbb{R}_{+}} G(u(x, 0), v(x, 0)) d x .
$$

We need only prove

$$
\int_{\mathbb{R}_{+}} G(u(x, 0), v(x, 0)) d x \geq 0 .
$$

To this end, we use Green's theorem for the quarter-plane $D=\{(x, y): x \geq 0$, $y \geq 0\}$, which gives

$$
\begin{aligned}
\int_{\mathbb{R}_{+}} G(u(x, 0), v(x, 0)) d x= & \iint_{D} y \Delta G(u(x, y), v(x, y)) d x d y \\
& +\left.\int_{0}^{\infty} y \frac{\partial G(u(x, y), v(x, y))}{\partial x}\right|_{x=0} d y .
\end{aligned}
$$

(In a similar situation, for the Hilbert transform on the real line, Green's theorem for the upper half-plane is applied in [E2].)

The above formula is easily justified using estimates (6.25) together with

$$
|G(z)| \leq C|z|^{p}, \quad|\partial G(z)| \leq C|z|^{p-1} .
$$

Note that the integrals over the positive real and imaginary axes in (6.31) are absolutely convergent, and the double integral is nonnegative since $G \circ \mathcal{F}(z)$ is subharmonic in the upper half-plane.

Let us show that the second integral on the right-hand side of (6.31) is nonnegative as well. Notice that

$$
\begin{aligned}
& \left.\frac{\partial G(u(x, y), v(x, y))}{\partial x}\right|_{x=0} \\
& =\frac{\partial G}{\partial x}(u(0, y), v(0, y)) \frac{\partial u}{\partial x}(0, y)+\frac{\partial G}{\partial y}(u(0, y), v(0, y)) \frac{\partial v}{\partial x}(0, y) .
\end{aligned}
$$

Using (6.28) and (6.29), we see that the first term on the right-hand side of the preceding equation vanishes, and

$$
\left.\frac{\partial G(u(x, y), v(x, y))}{\partial x}\right|_{x=0}=-\frac{\partial G}{\partial y}(\mathcal{L} f(y), 0) \frac{d(\mathcal{L} f)}{d y}(y) .
$$

Thus,

$$
\left.\int_{0}^{\infty} y \frac{\partial G((u(x, y), v(x, y))}{\partial x}\right|_{x=0} d y=-\int_{0}^{\infty} y \frac{\partial G}{\partial y}(\mathcal{L} f(y), 0) \frac{d(\mathcal{L} f)}{d y}(y) d y .
$$

As was established in the proof of Lemma 4.3, for $p \geq 2$, the function $\Phi$ defined by (6.27) coincides with its subharmonic minorant constructed 
above: $G(x, y)=\Phi(x, y)$ in some double sector containing the real axis. Hence

$$
\frac{\partial G}{\partial y}(x, 0)=\frac{\partial \Phi}{\partial y}(x, 0)=p x|x|^{p-2} .
$$

From this using integration by parts we obtain

$$
\begin{aligned}
& \left.\int_{0}^{\infty} y \frac{\partial G(u(x, y), v(x, y))}{\partial x}\right|_{x=0} d y \\
& =-p \int_{0}^{\infty} y \mathcal{L} f(y)|\mathcal{L} f(y)|^{p-2} \frac{d(\mathcal{L} f)}{d y}(y) d y=\int_{0}^{\infty}|\mathcal{L} f(y)|^{p} d y \geq 0
\end{aligned}
$$

which yields (6.30) for $p \geq 2$.

We now prove an analogous inequality which involves the adjoint operator $\Pi^{*}=\mathcal{F}_{\mathrm{c}} \mathcal{F}_{\mathrm{s}}$ :

$$
\left\|I-\Pi^{*}\right\|_{L^{p}\left(\mathbb{R}_{+}\right)} \leq B_{p}^{1 / p}, \quad p \geq 2
$$

or equivalently,

$$
\left\|\mathcal{F}_{\mathrm{c}} f-\mathcal{F}_{\mathrm{s}} f\right\|_{L^{p}\left(\mathbb{R}_{+}\right)}^{p} \leq B_{p}\left\|\mathcal{F}_{\mathrm{s}} f\right\|_{L^{p}\left(\mathbb{R}_{+}\right)}^{p}, \quad p \geq 2 .
$$

We set $H(x, y)=G(y, x)$, where $G$ is the subharmonic function used in (6.26). Then

$$
|x-y|^{p} \leq B_{p}|y|^{p}-H(x, y), \quad p \geq 2 .
$$

Obviously, $H(x, y)$ is a subharmonic minorant for

$$
\Psi(x, y)=B_{p}|y|^{p}-|x-y|^{p} .
$$

However,

$$
\Delta \Psi(x, y)=p(p-1)\left(B_{p}|y|^{p-2}-2|x-y|^{p-2}\right),
$$

from which it is easily seen that $\Psi(x, y)$ is superharmonic in a double sector $S$ containing the real axis. Consequently, by the construction of the subharmonic minorant in the proof of Theorem 3.7, $H(x, y)$ is harmonic in $S$, and $H(x, y)<\Psi(x, y)$ there.

We proceed using the fact that, in polar coordinates, $H$ can be expressed in the form

$$
H\left(r e^{i t}\right)=r^{p} h(t), \quad-\pi \leq t \leq \pi,
$$

where $h(t)$ is $\pi$-periodic since $\Psi(x, y)=\Psi(-x,-y)$.

Our next step is to show that (6.33) holds provided $h^{\prime}(0) \geq 0$. Arguing as above, we get

$$
\int_{\mathbb{R}_{+}}\left|\mathcal{F}_{\mathrm{c}} f(x)-\mathcal{F}_{\mathrm{s}} f(x)\right|^{p} d x \leq B_{p} \int_{\mathbb{R}_{+}}\left|\mathcal{F}_{\mathrm{s}} f(x)\right|^{p} d x-\int_{\mathbb{R}_{+}} H(u(x, 0), v(x, 0)) d x,
$$


where by Green's theorem

$$
\begin{aligned}
& \int_{\mathbb{R}_{+}} H(u(x, 0), v(x, 0)) d x \\
& \quad=\iint_{D} y \Delta H(u(x, y), v(x, y)) d x d y-\int_{0}^{\infty} y \frac{\partial H}{\partial y}(\mathcal{L} f(y), 0) \frac{d(\mathcal{L} f)}{d y}(y) d y .
\end{aligned}
$$

Since $H(u(x, y), v(x, y))$ is subharmonic, it remains to verify the inequality

$$
\int_{0}^{\infty} y \frac{\partial H}{\partial y}(\mathcal{L} f(y), 0) \frac{d(\mathcal{L} f)}{d y}(y) d y \leq 0
$$

Clearly,

$$
\frac{\partial H}{\partial y}=\frac{\partial H}{\partial r} \sin t+\frac{1}{r} \frac{\partial H}{\partial t} \cos t=r^{p-1}\left(h^{\prime}(t) \cos t+p h(t) \sin t\right),
$$

and using the fact that by $\pi$-periodicity $h^{\prime}( \pm \pi)=h^{\prime}(0)$, we obtain

$$
\frac{\partial H}{\partial y}\left(r e^{i t}\right)= \begin{cases}r^{p-1} h^{\prime}(0) & \text { if } t=0 \\ -r^{p-1} h^{\prime}(0) & \text { if } t= \pm \pi\end{cases}
$$

In other words, on the real axis

$$
\frac{\partial H}{\partial y}(x, 0)=x|x|^{p-2} h^{\prime}(0) \text {. }
$$

From this, using integration by parts as above, we conclude that

$$
\begin{aligned}
& \int_{0}^{\infty} y \frac{\partial H}{\partial y}(\mathcal{L} f(y), 0) \frac{d(\mathcal{L} f)}{d y}(y) d y \\
& \quad=h^{\prime}(0) \int_{0}^{\infty} y \mathcal{L} f(y)|\mathcal{L} f(y)|^{p-2} \frac{d(\mathcal{L} f)}{d y}(y) d y=-\frac{h^{\prime}(0)}{p} \int_{0}^{\infty}|\mathcal{L} f(y)|^{p} d y .
\end{aligned}
$$

Thus, (6.36) holds provided $h^{\prime}(0) \geq 0$.

We now prove in a rather indirect way that indeed $h^{\prime}(0) \geq 0$. (This is easily verified numerically, but a direct analytical proof seems to be tedious.) Observe that an analogous argument applied to $\left\|I+\Pi^{*}\right\|_{L^{p}\left(\mathbb{R}_{+}\right)}$in place of $\left\|I-\Pi^{*}\right\|_{L^{p}\left(\mathbb{R}_{+}\right)}$would boil down to the inequality

$$
|x+y|^{p} \leq B_{p}|y|^{p}-H(x,-y), \quad p \geq 2,
$$

which follows by changing $z$ to $\bar{z}$ in (6.34). In other words, one has to replace $h(-t)$ with $h(t)$, and consequently change $h^{\prime}(0) \geq 0$ to $h^{\prime}(0) \leq 0$.

The latter assumption, as was demonstrated above, would lead to the inequality

$$
\left\|I+\Pi^{*}\right\|_{L^{p}\left(\mathbb{R}_{+}\right)} \leq A_{p}=B_{p}^{1 / p}
$$


However, this contradicts Lemma 6.4 where it was shown using spectral theory that

$$
\left\|I+\Pi^{*}\right\|_{L^{p}\left(\mathbb{R}_{+}\right)}=1+\cot \frac{\pi}{2 p}, \quad p \geq 2 .
$$

It remains to notice that

$$
1+\cot \frac{\pi}{2 p}>A_{p}, \quad p \geq 2 .
$$

This inequality can be deduced from the following lemma which must be well known and whose proof we include for the sake of completeness.

Lemma 6.6. Suppose $T$ is a linear bounded operator on a uniformly convex Banach space $X$. If $\|I+T\|_{X}=1+\|T\|_{X}$ then $\|T\|_{X} \in \sigma(T)$ where $\sigma(T)$ denotes the spectrum of $X$.

Proof. If $\|I+T\|_{X}=1+\|T\|_{X}$ then clearly $\|I+t T\|_{X}=1+t\|T\|_{X}$ for every $t>0$, so without loss of generality we may assume that $\|T\|_{X}=1$.

If $X$ is uniformly convex then the modulus of convexity $\delta_{X}(\varepsilon)$ is positive for every $0<\varepsilon \leq 2$, where $\delta_{X}(\varepsilon)$ is defined by (see [LT, Sec. 1.e])

$$
\delta_{X}(\varepsilon)=\inf \{1-\|x+y\| / 2: x, y \in X,\|x\| \leq 1,\|y\| \leq 1,\|x-y\| \geq \varepsilon\} .
$$

Since $\|I+T\|_{X}=2$, we can choose $\alpha>0$ so that $\alpha<2 \delta_{X}(\varepsilon)$, and find $x \in X$ such that $\|x\|=1$ and $\|x+T x\| \geq 2-\alpha$. Then letting $y=T x$, so that $\|y\| \leq 1$, we deduce that $\|x-T x\|<\varepsilon$, since otherwise

$$
\alpha / 2<\delta_{X}(\varepsilon) \leq 1-\|x+T x\| / 2,
$$

which contradicts the estimate $\|x+T x\| \geq 2-\alpha$. Thus, for an arbitrarily small $\varepsilon>0$ there exists $x \in X$ such that $\|x\|=1$ and $\|x-T x\|<\varepsilon$, so $1 \in \sigma(T)$.

Now by Theorem 4.1, $A_{p}=\left\|I+H^{\mathbb{R}}\right\|_{L^{p}(\mathbb{R})}$ and $1+\cot \frac{\pi}{2 p}=1+\left\|H^{\mathbb{R}}\right\|_{L^{p}(\mathbb{R})}$, so by Lemma $6.6,1+\cot \frac{\pi}{2 p}=A_{p}$ would imply $\left\|H^{\mathbb{R}}\right\|_{L^{p}(\mathbb{R})} \in \sigma\left(H^{\mathbb{R}}\right)$. However, $\sigma\left(H^{\mathbb{R}}\right)=\{ \pm i\}$ on $L^{p}(\mathbb{R}), 1<p<\infty$, which proves that (6.38) holds.

The proof of (6.36) is complete. This proves (6.33), and hence (6.20) for $p \geq 2$, while the case $1<p \leq 2$ follows by duality.

\section{References}

[Ba] A. Baernstein, II, The *-function in complex analysis, in: Handbook of Complex Analysis, Vol. I: Geometric Function Theory, R. Kühnau (ed.), North-Holland, Amsterdam, in preparation.

[Be] W. Beckner, Inequalities in Fourier analysis, Ann. of Math. (2) 102 (1975), 159182.

[BG] E. Berkson and T. A. Gillespie, The generalized M. Riesz theorem and transference, Pacific J. Math. 120 (1985), 279-288. 
[Bir] M. S. Birman, Re-expansion operators as objects of spectral analysis, in: Linear and Complex Analysis Problem Book, Lecture Notes in Math. 1043, Springer, 1984, 130-134.

[Bur] D. L. Burkholder, Martingales and singular integrals in Banach spaces, in: Handbook of the Geometry of Banach Spaces, Vol. I, W. B. Johnson and J. Lindenstrauss (eds.), North-Holland, Amsterdam, 2001, 233-269.

[E1] M. Essén, A superharmonic proof of the M. Riesz conjugate function theorem, Ark. Mat. 22 (1984), 241-249.

[E2] - Some best constant inequalities for conjugate functions, in: General Inequalities 6 (Oberwolfach, 1990), Internat. Ser. Numer. Math. 103, Birkhäuser, Basel, 1992, 129-140.

[ESS] M. Essén, D. F. Shea, and C. S. Stanton, Sharp $L \log ^{\alpha} L$-inequalities for conjugate functions, Ann. Inst. Fourier (Grenoble) 52 (2002), 623-659.

[G] T. W. Gamelin, Uniform Algebras and Jensen Measures, London Math. Soc. Lecture Note Ser. 32, Cambridge Univ. Press, Cambridge, 1978.

[GK1] I. Gohberg and N. Krupnik, Norm of the Hilbert transformation in the $L_{p}$ space, Funktsional. Anal. i Prilozhen. 2 (1968), no. 2, 91-92 (in Russian); English transl.: Functional Anal. Appl. 2 (1968), 180-181.

[GK2] - - -, One-Dimensional Linear Singular Integral Equations, Vols. I, II, Oper. Theory Adv. Appl. 53, 54, Birkhäuser, Basel, 1992.

$[\mathrm{HV}]$ B. Hollenbeck and I. E. Verbitsky, Best constants for the Riesz projection, J. Funct. Anal. 175 (2000), 370-392.

[I1] E. M. Il'in, Scattering characteristics of a problem of diffraction by a wedge and by a screen, Zap. Nauchn. Sem. LOMI 107 (1982), 193-197 (in Russian); English transl.: J. Soviet Math. 36 (1987), 417-419.

[I2] - The scattering matrix for a problem of diffraction by a wedge, in: Operator Theory and Function Theory, No. 1, Leningrad Univ., 1983, 87-100 (in Russian).

[Ka] N. J. Kalton, Plurisubharmonic functions on quasi-Banach spaces, Studia Math. 84 (1986), 297-324.

[K] N. Krupnik, Banach Algebras with Symbol and Singular Integral Operators, Oper. Theory Adv. Appl. 26, Birkhäuser, Basel, 1987.

[L1] B. Ya. Levin, Distribution of Zeros of Entire Functions, Transl. Math. Monogr. 5, Amer. Math. Soc., Providence, RI, 1964.

[L2] - Lectures on Entire Functions, Transl. Math. Monogr., 150, Amer. Math. Soc., Providence, RI, 1996.

[Li] E. H. Lieb, Gaussian kernels have only Gaussian maximizers, Invent. Math. 102 (1990), 179-208.

[LT] J. Lindenstrauss and L. Tzafriri, Classical Banach Spaces, II, Springer, Berlin, 1979 .

[N] N. K. Nikol'skiü, Treatise on the Shift Operator. Spectral Function Theory, Springer, Berlin, 1986.

[Pe] A. Pełczyński, Norms of classical operators in function spaces, Colloque en l'honneur de Laurent Schwartz, Vol. I, Astérisque 131 (1985), 137-162.

[Pi] S. K. Pichorides, On the best values of the constants in the theorems of M. Riesz, Zygmund and Kolmogorov, Studia Math. 44 (1972), 165-179.

[Po] E. A. Poletsky, Plurisubharmonic functions as solutions of variational problems, in: Several Complex Variables and Complex Geometry, Part I, Proc. Sympos. Pure Math. 52, Amer. Math. Soc., Providence, RI, 1991, 163-171.

[St] E. M. Stein, Harmonic Analysis: Real-Variable Methods, Orthogonality, and Oscillatory Integrals, Princeton Univ. Press, Princeton, NJ, 1993. 
[V] I. E. Verbitsky, An estimate of the norm of a function in a Hardy space in terms of the norms of its real and imaginary parts, Mat. Issled. 54 (1980), 16-20 (in Russian); English transl.: Amer. Math. Soc. Transl. (2) 124 (1984), 11-15.

[Z] A. Zygmund, Trigonometric Series, Vol. 2, Cambridge Univ. Press, London, 1968.

Department of Mathematics

Emporia State University

Emporia, KS 66801, U.S.A.

E-mail: hollenbeb@esumail.emporia.edu
Department of Mathematics University of Missouri Columbia, MO 65211, U.S.A. E-mail: nigel@math.missouri.edu igor@math.missouri.edu

Received January 15, 2002

Revised version March 14, 2003 